\title{
Acceptance of matchmaking tools in coworking spaces: an extended perspective
}

\section{Cristopher Siegfried Kopplin ${ }^{1}$ (D) Till Marius Gantert ${ }^{2} \cdot$ Julia Verena Maier $^{3}$}

Received: 31 December 2020 / Accepted: 24 September 2021 / Published online: 11 October 2021

(c) The Author(s) 2021

\begin{abstract}
An extended technology acceptance model for matchmaking tools in coworking spaces is presented and tested among 92 German coworkers. Advancing previous research, hedonic and community-related aspects are integrated into a framework based on the extended unified theory of acceptance and use of technology (UTAUT2). Coworkers emphasize a matchmaking tool's productivity aspects, which are positively moderated by their sense of community. Hedonic motivation (HM) and personal innovativeness contribute to usage intention, while effort expectancy is not a driver. The influence of HM is negatively moderated by sense of community, suggesting that a favorable social atmosphere that is explorable in person acts as a partial substitute for the enjoyment of tool usage. Surprisingly, satisfaction with face-to-face activities does not affect their perception of a matchmaking tool.
\end{abstract}

Keywords Coworking spaces · Matchmaking tools · Sense of community · Technology acceptance $\cdot$ Partial least squares $\cdot$ Structural equation modeling

JEL Classification M13 · M19 - O30 - L26

Cristopher Siegfried Kopplin

cristopher.kopplin@uni-bayreuth.de

Till Marius Gantert

till1.gantert@uni-bayreuth.de

Julia Verena Maier

julia.maier@uni-bayreuth.de

1 Chair of Marketing and Innovation, Universitätsstraße 30, 95447 Bayreuth, Germany

2 Chair of Strategic Management and Organization, Prieserstrasse 2, 95447 Bayreuth, Germany

3 University of Bayreuth, Universitätsstraße 30, 95447 Bayreuth, Germany 


\section{Introduction}

Coworking spaces (CWS) are membership-based innovation hubs for cross-fertilization, inspiration, professional exchange, and economic environments that provide essential infrastructure for daily business (Bouncken and Reuschl 2018; Garrett et al. 2017; Gerdenitsch et al. 2016). A growing body of evidence indicates that interaction indeed yields positive impacts on coworkers, such as enhancing knowledge sharing and creative performance (Rese et al. 2020) and increasing work satisfaction (Bouncken et al. 2020a). Even more important, evidence has been found that ascribes the community a critical role in deciding to stay in or leave a CWS (Garrett et al. 2017), and nascent coworkers highly profit from more experienced members that may act similar to mentors (Bouncken and Aslam 2019). On the other hand, research also emphasizes that CWS are fragile business models (Seo et al. 2017). As such, providers need to understand their coworkers' needs and demands and offer a portfolio of adequate solutions, such as creating a creative and supportive environment, reinforced by infrastructure and events (e.g., workshops) (Blagoev et al. 2019; Garrett et al. 2017). One of the main challenges, therefore, is understanding, inducing, and managing social interaction among coworkers.

For this undertaking, a holistic perspective, including both physical and digital amenities as well as coworkers' perception of these, is deemed necessary to investigate coworking-related phenomena. As digital applications are an inevitable component of a CWS (Bouncken et al. 2020b), these software tools need to be included in CWS' design, amelioration, and management. Recent findings have shown that CWS are environments of Co-Prosumption, i.e., co-located coworkers cocreate value with the CWS provider in the same service space (Bouncken and Tiberius 2021). Hence, the software infrastructure also influences value co-creation and, ultimately, impacts the CWS' service space manifestation: coworkers may coordinate cocreation among themselves, which may be affected by technology, work on their own, or combine both (Bouncken and Aslam 2019; Spinuzzi 2012; Spinuzzi et al. 2019). An example of software-based coordination lies in so-called innovation management software, which is used in companies and ecosystems to emphasize the aspects of innovation management in the innovation process (e.g., Huesig and Endres 2019; Endres et al. 2021). Contrary to companies, professionals in CWS first need to find each other to use appropriate software for better coordination.

Previous research has outlined the concept of so-called matchmaking tools, which are software applications meant to facilitate social connections and, as such, elicit serendipity, creativity, and innovation (Kopplin 2020). Matchmaking tools offer a platform that serves as an intermediary between physical and digital, i.e., online and offline, activities within the CWS and increases the number of possible business opportunities and the probability of encountering serendipitous situations, which some authors have proposed the main principle of CWS (Moriset 2013). For example, they may link individuals from different backgrounds who may profit from a de-contextualization, i.e., an idea's transfer from one realm 
to another (Bouncken and Aslam 2019), which in essence is a coordination problem. Through their algorithmic nature, in contrast to more or less casual, free-ofconstraints face-to-face encounters, they may take the problem of pre-discoveries into account. This term refers to serendipitous insights that cannot be exploited as they are not recognized due to a lack of knowledge or skill (Merton and Barber 2004). By creating digital profiles that may be specified with rich data on professional experience, personal interest, and existing knowledge, matchmaking tools can introduce coworkers who are likely to benefit from the encounter.

For the applications to manifest their benefits, it is critical that coworkers make use of them. Thus, their technology acceptance needs to be ensured. First insights into coworkers' acceptance of matchmaking tools have been presented by Kopplin (2020), with a focus on matchmaking tools conceptual layout and their utilitarian benefits. To understand the prospects and the role of these applications, it is necessary to integrate them within a broader nomological net extending the utilitarian view, which considers hedonic factors as well as CWS-specific contextual influences such as the availability of "offline" activities such as workshops, breakfasts, and pitch sessions, which are common elements of coworking (Blagoev et al. 2019). Thus far, little research has investigated the acceptance of matchmaking tools in CWS.

In a nutshell, the extant work on matchmaking tools is somewhat theoretical and needs empirical amplification. Besides the study by Kopplin (2020), insights are scarce. The purpose of this study is to advance our understanding of these applications in two substantial ways: first, additional empirical evidence for the impact of utilitarian aspects is provided, and the research perspective is extended to further include hedonic and context-dependent variables, namely sense of community and coworkers' satisfaction with offline activities. Second, the influence of user perceptions on technology acceptance is assessed from sufficiency and necessity angles, thus presenting 'both sides of the coin'. In total, we provide a contextualized and methodologically holistic view of coworkers' acceptance of matchmaking tools. Thus, our research questions are as follows:

RQ1: Which influence factors drive coworkers' acceptance of matchmaking tools?

RQ2: What is the nature of this influence in terms of necessity and sufficiency?

Based on a UTAUT2 framework, an adapted model is derived and tested using partial least squares structural equation modeling (PLS-SEM), complemented by a necessary condition analysis (NCA) (Dul 2016a). Our study contributes to the CWS literature by identifying the structure and impact of coworkers' perception of matchmaking tools on their intended usage behavior, providing insights into their goals when using such an application, and offering starting points for CWS providers' deliberate design of the CWS' technological infrastructure. By contextualizing a UTAUT2-based model, we also contribute to the technology acceptance literature as we demonstrate the implementation of variables capturing the social surrounding as well as alternatives that may serve the same purposes as the technology under investigation.

The remainder of the paper is structured as follows. Section 2 outlines the related work and sheds light on the theoretical underpinnings. The research design is 
presented in Sect. 3, followed by the results in Sect. 4, and a discussion in Sect. 5. The final section gives concluding remarks and provides an outlook for future research.

\section{Theoretical background}

\subsection{Role and acceptance of technology in coworking spaces}

CWS have mushroomed worldwide and sparked a rich body of scientific studies from a broad range of disciplines. As out-of-the-box workplaces, they offer a mixture of basic infrastructure, such as Internet access, and various amenities like workshops (Spinuzzi 2012). Coworking spaces (CWS) are membership-based innovation hubs for cross-fertilization, inspiration, and professional exchange, and economic environments that provide essential infrastructure for daily business (Bouncken and Reuschl 2018; Garrett et al. 2017; Gerdenitsch et al. 2016). Individuals as well as startup companies and employees of large corporations work alongside each other and utilize the same physical and digital amenities (Bianchi et al. 2018), which may comprise basic technology such as WiFi access, but also social events in the form of pitch sessions, workshops, and the like (Blagoev et al. 2019; Garrett et al. 2017). Within this environment, coworkers have personal goods (e.g., a mailbox or a messaging inbox) and collective goods (e.g., mutually used lounge areas, phone booths) at their disposal (DeGuzman and Tang 2011). In short, CWS may be described as surroundings of sociomateriality (Bouncken et al. 2020b; Orlikowski 2007) that offer an out-of-the-box ecosystem.

This interplay between a community of like-minded people and supporting infrastructure renders interaction among coworkers a vital component of everyday coworking. The extant literature has found mixed evidence regarding CWS' community spirit, and the phrase 'working alone together' has been cited in many publications (coined by Spinuzzi 2012; see also the discussion of the term community in Spinuzzi et al. 2019). As Bouncken et al. (2020b) summarize, coworking has not lived up to expectations so far and has brought disappointment on the sides of providers and coworkers. It appears that mere spatial proximity is not sufficient for beneficial interaction; instead, cognitive proximity needs to be created (Bouncken and Aslam 2019; Kopplin 2020). As one remedy, digital tools for information dissemination and supporting coworkers find complementary resources, such as a business partner, have been proposed (Bouncken et al. 2020b). The study at hand hence investigates drivers of coworkers' technology acceptance of matchmaking tools to understand drivers and barriers of their use and advances our current knowledge by integrating hedonic as well as environmental factors in terms of coworkers' sense of community and the availability of potentially competitive 'offline' activities such as pitch sessions and workshops. In this vein, we adapt the extant knowledge about technology acceptance to reflect coworking's characteristics and provide a coworking-specific conceptual framework. Further, we provide empirical data on coworkers' evaluation of potential uses regarding matchmaking tools in their daily routine. 
For CWS providers to successfully implement such an application, it is critical to understand coworkers' perceptions and evaluations of this type of software.

\subsection{Utilitarian beliefs}

Due to their embedding in the sharing economy (Bouncken et al. 2020a; Gandini 2015), CWS are characterized by the idea of an interactive environment that enables collaboration (Schmidt and Brinks 2017). This sharing focus is enabled by the omnipresence and availability of digitalized tools, making it feasible to connect supply and demand anytime and anywhere, and, thus, it emphasizes the role of access to resources instead of owning them (Belk 2014). Recent research indicates that collaborative efforts, such as knowledge sharing, increase coworkers' creative performance, which is frequently viewed as a major benefit of CWS (Bouncken and Aslam 2019; Parrino 2015; Rese et al. 2020). Indeed, a thrive to learn has been proposed as the defining quality of coworking (Bouncken and Aslam 2019; Waters-Lynch and Potts 2017). Seo et al. (2017) add that CWS provide an essential realm for exchange and gaining experience, which is of particular help in regions with high numbers of one-person companies and startups. This functionality of providing a junction, serving as a beacon for those who need it, appears vastly similar to the Schelling Point notion mentioned by Waters-Lynch and Potts (2017). In essence, CWS may be described as loci for conjointly venturing, which implies coordination requirements among coworkers.

Within a typical CWS, several mechanisms are in place to facilitate socially connecting for inspiration and innovation. Common elements comprise digital member directories, newsletters, and social events, but also community hosts, and digital interfaces between the digital and physical realm such as matchmaking tools (Kopplin 2020; Waters-Lynch and Potts 2017). Coworkers, of course, need to be aware of these amenities to be able to benefit from them. Such learning opportunities that have been institutionalized "support the combination and recombination of knowledge" (Bouncken and Aslam 2019), and, consequently, are valuable assets for both coworkers and CWS providers. Focusing on mere co-location for collaboration can be misleading (Spinuzzi 2012), as instead, the interplay of "social actors and material artifacts" (Bouncken and Aslam 2019) needs to be considered. Any solution that seeks acceptance thus needs to bear an advantage regarding identifying and approaching helpful others, i.e., coworkers will evaluate whether it is reasonable to assume "the existence of a use-performance relationship" (Agarwal and Karahanna 2000). As CWS are spheres where the online and the offline realms mix, and coworkers may ask around for help in person, use a bulletin board, or ask the community host for information, it is assumed that rational decision-makers will also evaluate whether the usage of a matchmaking tool is low in effort. Hence, we derive the following hypotheses: 
H1 Performance expectancy (PE) has a positive impact on coworkers' behavioral intention to use a matchmaking tool.

H2 Effort expectancy (EE) has a positive impact on coworkers' behavioral intention to use a matchmaking tool. ${ }^{1}$

\subsection{Hedonic beliefs and personal innovativeness}

Recent years have witnessed the implementation of technologies previously known from consumer settings in the professional context. This phenomenon has been termed consumerization and proposes to view individuals as consumers, regardless of whether they are in an actual demand situation or on the supply side (Harris et al. 2012). We believe that consumerization adequately represents the entanglement of coworkers within a CWS, and, particularly in the case of matchmaking tools, it is likely that most coworkers have experiences with similar applications from their private contexts. For example, Tinder may be a prominent specimen of matchmaking tools in the realm of romantic relationships.

Further, including private settings into the professional context renders coworkers' behavioral beliefs about receiving hedonic benefits an essential factor (Venkatesh et al. 2012). This integration is also consistent with the notion of dual-purpose information systems, i.e., applications that fulfill both utilitarian and hedonic needs, and of which communication-related tools are a prominent example (Wu and Lu 2013). Thus, we hypothesize:

H3 Hedonic motivation has a positive impact on coworkers' behavioral intention to use a matchmaking tool.

Extant research has further proposed to include the concept of personal innovativeness (PI) (Kopplin 2020). As CWS yield an innovative, creative, and inspirational nature, coworkers may feel attracted to novel tools that incorporate a progressive and forward-looking quality. PI has been introduced to the literature to explain why some individuals are willing to employ a new technology while others are not, and has a long history in research on innovation diffusion but also marketing (Agarwal and Prasad 1998). In the literature on the diffusion of innovations, notions of consumers being located on a continuum from innovative to not innovative serve as anchor points for segmentation based on the time of particular individuals' adoption (Rogers and Shoemaker 1971). This conceptualization has not been without criticism (see, e.g., Midgley and Dowling 1978), and more specific definitions distinguishing between global and domain-specific innovativeness have been proposed (Flynn and Goldsmith 1993). Consistent with this distinction, Agarwal and Prasad (1998) develop the concept of PI in the domain of information technology and view

\footnotetext{
1 Note that in the technology acceptance field, EE is commonly reverse scaled (e.g., in UTAUT2), i.e., high values indicate perceptions of low effort.
} 
it as an essential moderator for technology acceptance measurement. However, this specification has led to mixed results, and Agarwal and Karahanna (2000) proposed a new construct termed cognitive absorption as an antecedent of TAM-based variables (i.e., perceived usefulness and perceived ease of use), which in turn is influenced by PI and playfulness. Here, a first shift from a moderating role towards an antecedent could be observed.

Complementary research shows that it may be adequately used as a factor of behavioral intention; however, it is mediated by TAM-related constructs such as perceived usefulness and perceived ease of use (Jackson et al. 2013; Lu et al. 2005), corroborating the work by Agarwal and Karahanna (2000). For example, Lu et al. (2005) argue that predominant technology acceptance models are commonly used after adoption, and, as such, they are more related to continuous use than to acceptance. They conclude that for the pre-adoption context, "holistic experiences with technology as captured in constructs such as enjoyment, flow, and social image are potentially important explanatory variables in technology acceptance" (Lu et al. 2005). During this initial phase of adoption, "decision-making is exposed to variables other than those incurred by the technology itself" (Lu et al. 2005). We need to understand how beliefs about a technology's properties are created (Agarwal and Karahanna 2000). Besides perceived relevance (PR), PI is included for this purpose. Consequently, we derive:

H4a Personal innovativeness has a positive impact on coworkers' behavioral intention to use a matchmaking tool.

H4b Personal innovativeness has a positive impact on hedonic motivation.

\subsection{Perceived relevance}

The extant literature mentions that coworkers may follow different goals and behavioral patterns during their visits. In the terminology provided by Bouncken et al. (2020a), they may be classified as utilizers (i.e., they focus on the infrastructure), socializers (i.e., social acknowledgment is emphasized), and learners (i.e., knowledge acquisition is the main motive). To incorporate this heterogeneity in our model, we propose that not only the perception that a matchmaking tool will support social interactions will have an impact, but also coworkers' belief that such tools' capabilities match their own goals. For example, a socializer may prefer attending events in person rather than assessing member profiles on her smartphone, i.e., a matchmaking tool's PR may be low. PR has been studied in a variety of contexts such as online advertising (Campbell and Wright 2008; Jung 2017), Internet use for information seeking (Shih 2004), media usage in education (Hu et al. 2003), and technology acceptance in general (Bhattacherjee and Sanford 2006), and describes individuals' impression of goal congruency, i.e., the object under investigation is "related to personal needs and values" (Jung 2017) and "instrumental in achieving their personal goals" (Celsi and Olson 1988). Extant research shows that PR has a positive influence on attitude, which is established by beliefs about the likely outcomes of 
a certain behavior (Celsi and Olson 1988; Fishbein and Ajzen 1975; Madden et al. 1992). Consequently, PR is expected to impact the perception of technology's performance capabilities positively.

When a coworker observes a high degree of congruency between personal goals and the matchmaking tool's capabilities, it is also assumed that the effort that is necessary to make use of the tool is viewed in a positive light, as a sort of investment that helps to take the next steps towards one's goal. Indeed, evidence has been found that notions of usefulness and ease of use are influenced by PR (Shih 2004). Further, depending on the individual coworker's goals, the entertaining, i.e., hedonic component of the application, may be the vital reason for usage. We thus hypothesize:

H5a Perceived relevance has a positive impact on performance expectancy.

H5b Perceived relevance has a positive impact on effort expectancy.

H5C Perceived relevance has a positive impact on hedonic motivation.

\subsection{Community and social interaction}

The final set of hypotheses includes coworkers' sense of community and their interactions in the physical rather than the digital realm. These context factors are critical to understanding the role of matchmaking tools in CWS, as, for example, a particular coworker's goals may be entirely achievable through personal communication, rendering a software application for the same purpose irrelevant. A CWS' community may be viewed as consisting of distinct economic entities-which may be individual freelancers, startup teams, and other actors such as employees from incumbent organizations-and consequently, there is commonly no shared economic agenda (Bianchi et al. 2018; Garrett et al. 2017). Hence, initiating a collaboration is unsolicited.

In this regard, it is important to keep in mind that CWS are not only practical, utility-focused environments but organizational phenomena (Blagoev et al. 2019), and the notion of community is central to these places (Bouncken et al. 2020a; Garrett et al. 2017; Gerdenitsch et al. 2016; Spinuzzi et al. 2019). In contrast to a neutral container " (Blagoev et al. 2019), CWS provide opportunities for networking and identification (Capdevila 2013). As Waters-Lynch and Potts (2017) mention, coworkers frequently do not seek traditional office standards but pay their membershipfee for entering a space that solves the coordination problem of "who needs to be met'. Against the backdrop of a matchmaking tool's capabilities, the concept of a coordination problem is a valuable theoretical lens. In line with Waters-Lynch and Potts (2017), we deem community and coordination not mutually exclusive perspectives on coworking but complements that provide a partial understanding each. Exchange is contingent upon social factors such as trust and the perceived absence of opportunity (Bouncken and Reuschl 2018; Rese et al. 2020; Spinuzzi et al. 2019). Consequently, a favorable assessment of a CWS' social sphere should positively affect coworkers' perception of a matchmaking tool. 
We use two constructs to capture the interplay of personal and digital interaction in CWS: sense of community and satisfaction with offline activities. Both constructs are assumed to mirror the social sphere of the CWS. The open layout and architecture has been mentioned as a facilitator for social interaction (Bilandzic and Foth 2013), and, as such, a certain degree of rivalry between online and offline activities may be expected.

We, therefore, hypothesize:

H6a Sense of community positively moderates the impact of performance expectancy on coworkers' behavioral intention to use a matchmaking tool.

H6b Sense of community positively moderates the impact of effort expectancy on coworkers' behavioral intention to use a matchmaking tool.

H6c Sense of community positively moderates the impact of hedonic motivation on coworkers' behavioral intention to use a matchmaking tool.

Considering the role of offline activities, we expect a moderating influence on PR's impact. PR, capturing the perception of congruency between the individual demands and the technology's capabilities, should be unaffected by the availability of alternatives, as this congruency is an absolute rather than a relative assessment and is not context-dependent. Thus, we conclude that satisfaction with offline activities should not be a factor of PR. The PR of the tool itself should not be altered by the mere presence of alternatives. Instead, it is expected that satisfaction with these alternatives will attenuate, i.e., negatively moderate, the impact that PR has on the coworker's perceptions of $\mathrm{PE}$ and EE: the more satisfied the individual coworker is regarding alternatives, the less substantial they may feel to use the tool due to a high degree of congruency between the application's capabilities and their job's demands, as alternatives may provide more attractive benefits (e.g., they may exhibit an even more substantial congruency). In other words, it is expected that the criticality of a goal congruency is diminished when other opportunities are available that may also help to achieve a certain goal. This leads to the following hypotheses:

H7a Satisfaction with offline activities negatively moderates the impact of perceived relevance on performance expectancy.

H7b Satisfaction with offline activities negatively moderates the impact of perceived relevance on effort expectancy.

Figure 1 displays the final research model. 


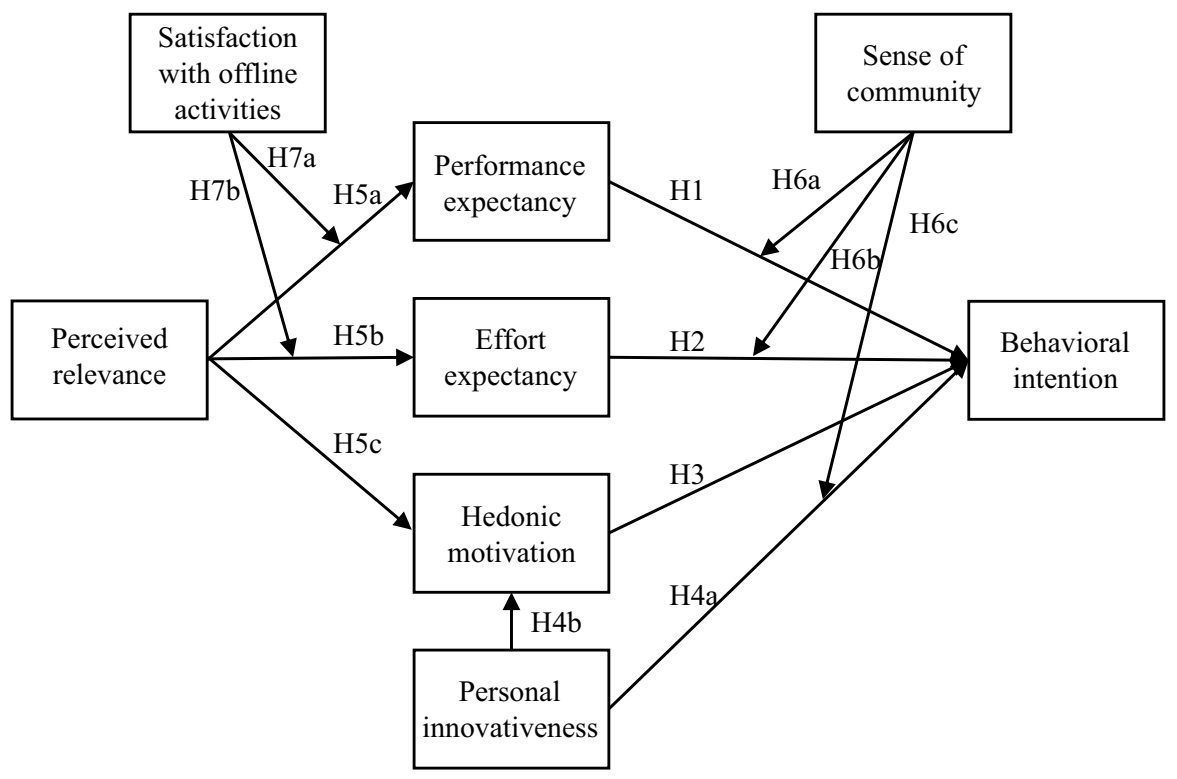

Fig. 1 Research model and hypotheses

\section{Research design}

\subsection{Constructs and items}

We adopted established measures from the literature. The items for the 'core model', i.e., PE, EE, and HM postulated to impact BI, are taken from the UTAUT2 framework (Venkatesh et al. 2012). PI is operationalized drawing on the indicators suggested by Agarwal and Prasad (1998). For PR, we adopted the construct from Alalwan (2018). SOA is measured with items from Liaw (2008) and Arbaugh (2000). Finally, we used the scale proposed by Peterson et al. (2008) for SOC. A complete list is provided in "Appendix A".

To contextualize our model, we modified the framework of UTAUT2 (Venkatesh et al. 2016). Compared to the original model, we omitted the variables social influence, facilitating conditions, habit, and price value (Venkatesh et al. 2012). Price value was dropped as currently introduced matchmaking tools are part of the CWS' infrastructure (Kopplin 2020) and do not require the payment of an additional fee. As such, this consideration would not reflect the actual situation within the CWS. Habit is a construct with an extensive literature stream and is discussed widely and inconsistently in the technology acceptance literature. Some scholars propose technology use models driven by habit as an automatism (Guinea and Markus 2009; Jasperson et al. 2005; Limayem et al. 2007). In the context of a novel technology, which applies to the study at hand, it appears inadequate to include habit, as many coworkers are expected to lack experience with these tools. Social influence, essentially capturing a form of peer pressure (Venkatesh et al. 2003, 2012), was swapped 
for the more detailed sense of community. We admit that the two variables are distinct; however, it is deemed more context relevant to include the particular notion of a sense of community. It is also expected that this sense is related to coworkers' belief of being able to find help and resources, conflicting with facilitating conditions (Venkatesh et al. 2012). Thus, these two UTAUT2 variables were removed from the final research model.

\subsection{Questionnaire design}

The questionnaire was drafted using Qualtrics. All constructs were measured on a five-point Likert-type scale, ranging from 'I completely disagree' (i.e., 1) to 'I completely agree' (i.e., 5). To prevent common method bias, participants were instructed that there were no incorrect answers, and the collected data will be handled confidentially. As an incentive to complete the survey, respondents had the opportunity to participate in a lottery and had the chance to win gift cards (25 Euros of value) for a large online shopping platform.

All items were adopted from the literature, as there was no need to draft new variables.

\subsection{Sampling strategy}

German CWS were targeted for sampling. Due to the different types of CWS emerging over time (Blagoev et al. 2019), it is worthwhile to address which kind of spaces we targeted briefly. In the terminology provided by Bouncken et al. (2018), our population may be classified as independent CWS. We did not include incumbent organizations that designed offices for their employees in the architecture of CWS, and we also excluded highly specialized hubs as we believe these would introduce a vast amount of heterogeneity due to potential (harmful) competition among the coworkers. Independent CWS, in contrast, are expected to yield coopetition, i.e., a duality of collaboration and competition, which is linked to innovation potential (Bouncken et al. 2018).

Using websites for desk booking, blogs, newspaper reports, and complementary search engine queries, an exhaustive list of 500 CWS located in Germany was compiled. Employing a cluster sampling approach, $340 \mathrm{CWS}$ were selected from that list and contacted via telephone and a follow-up e-mail, including a link to the survey. Each sampled CWS was asked to provide five completed questionnaires from coworkers.

\section{Results}

\subsection{Descriptive statistics}

In total, 182 questionnaires were received from $43 \mathrm{CWS}$, of which 101 were complete and considered for data assessment. This divergence may be partially explained 
by our sampling strategy: CWS managers were asked to spread the questionnaire, and they opened the survey to gain insights into its content before passing it on to the coworkers. Considering incomplete questionnaires that moved beyond the first three questions only (i.e., the first page yielding questions), we report a completion rate of $78.9 \%$. To ensure high data quality, the received questionnaires are checked for speeders and straightliners. Three data points had to be removed due to unlikely short response times, and five more observations were deleted because of strong indication for straightliners (three showed extreme response behavior, and the remaining two selected indifferent answers for all questions). Another data point was classified as a slower (i.e., the opposite of a speeder), who had a response time exceeding $24 \mathrm{~h}$, and was removed. In total, 92 questionnaires qualified for analysis. "Appendix E" lists the CWS, their location, and the gathered responses. As can be seen, most questionnaires stem from major cities such as Berlin, Munich, Hamburg, and Dusseldorf.

Regarding age, our sample is consistent with earlier reports on coworkers, with a mean age of 34.63 years (median $=33$ ) and a standard deviation of 7.79. The femaleto-male ratio was balanced, with $47.4 \%$ women and $52.6 \%$ men. In line with the extant literature, which noticed a shift from freelancers towards employees, only $14.7 \%$ reported they worked as freelancers, while 52.6\% were employees. About a sixth $(16.8 \%)$ was entrepreneurs and $10.5 \%$ employers. Concerning industries, most respondents are rooted in the IT field $(28.4 \%)$, followed by consulting (17.9\%), management $(11.6 \%)$, and marketing $(8.4 \%)$.

We also collected data about the CWS' social spheres. Table 1 provides an overview of coworker and CWS characteristics. The largest proportion of coworkers visits the space five times a week, followed by a group coming in four days. Predominant CWS sizes in terms of members were small (less than ten members) and medium (11-30 members). Membership type varied, with similar proportions of coworkers renting hot desks, fixed desks, and individual rooms. A small fraction (4.2\%) indicated other modes of use, such as a mixture of fixed desks and rooms. Regarding accessibility, most CWS allow 24/7 coworking (73.7\%), while some have regular office hours $(20.0 \%)$. A minority of spaces yields other access modes, such as distinguishing workdays from weekends and providing different office hours $(6.3 \%)$.

Asked for offline activities, i.e., social events such as breakfasts, workshops, and pitch sessions, $76.8 \%$ responded their CWS offers this type of amenity. $10.5 \%$ refused, while $12.6 \%$ indicated that they did not know. Consequently, matchmaking tools may be assumed to be deployed in an environment requiring integrating both the digital and the analog realm. Consistent with previous research, matchmaking tools are considered relatively new technology and most coworkers have not used such an application before (95.8\%). After being introduced to the stateof-the-art and typical features, as described in Kopplin (2020), we asked the participants to assess possible use scenarios of the tool. Two-thirds $(64.2 \%)$ responded they would seek to get in touch with fellow coworkers, and $62.1 \%$ would look for support with current challenges. About half of the participants $(51.6 \%)$ mentioned finding learning opportunities, and $44.2 \%$ would search collaboration partners for a project idea. Coworkers also stated more business-related applications: identifying 
Table 1 Coworker and CWS characteristics
Characteristic

Response

frequency

(\%)

\section{Visit frequency}

Five days a week

31.6

Four days a week

16.8

Three days a week

14.7

Twice a week

11.6

Once a week 8.4

Less frequent than once a week $\quad 10.5$

CWS size

Less than 10 coworkers $\quad 35.8$

11 to 30 coworkers $\quad 33.7$

31 to 40 coworkers $\quad 20.9$

More than 40 coworkers $\quad 9.6$

Membership modality

Hot desk 29.5

Fixed desk $\quad 30.5$

Individual room $\quad 35.8$

Other $\quad 4.2$

CWS accessibility

$\begin{array}{ll}24 / 7 & 73.7\end{array}$

Office hours $\quad 20.0$

Other 6.3

Membership duration

More than one year 37.9

6 to 12 months $\quad 17.9$

3 to 5 months $\quad 20.0$

Less than 3 months $\quad 24.2$

Future membership plans

Stay for more than one year $\quad 60.0$

Stay for 6 to 12 months $\quad 17.9$

Stay for less than 6 months $\quad 22.1$

Coworker typology

Socializer $\quad 63.2$

Utilizer $\quad 62.1$

Learner $\quad 7.4$

Coworker typology was ascribed due to self-reported main motives. Multiple responses were possible

new customers (46.3\%), expanding their professional network (67.4\%), and finding partners for the incorporation of an enterprise (7.4\%). Altogether, most coworkers identified as either socializers or utilizers instead of learners; however, the majority still emphasizes matchmaking tools' potential for identifying learning opportunities. 
Table 2 Construct assessment

\begin{tabular}{|c|c|c|c|c|c|}
\hline & Indicators & Mean (SD) & Cronbach's alpha & $\begin{array}{l}\text { Composite reli- } \\
\text { ability }\end{array}$ & AVE \\
\hline PE & 4 & $4.49(1.24)$ & 0.931 & 0.951 & 0.828 \\
\hline $\mathrm{EE}$ & 4 & $5.39(0.92)$ & 0.834 & 0.889 & 0.666 \\
\hline $\mathrm{HM}$ & 3 & $4.85(1.08)$ & 0.865 & 0.916 & 0.785 \\
\hline PI & 4 & $4.91(1.22)$ & 0.820 & 0.877 & 0.648 \\
\hline BI & 4 & $4.49(1.23)$ & 0.899 & 0.930 & 0.770 \\
\hline PR & 4 & $4.41(1.40)$ & 0.942 & 0.959 & 0.853 \\
\hline SOC & 4 & $5.31(1.22)$ & 0.877 & 0.899 & 0.695 \\
\hline SOA & 3 & $5.27(0.89)$ & 0.754 & 0.840 & 0.641 \\
\hline
\end{tabular}

$\mathrm{AVE}=$ average variance extracted

\subsection{Outer model evaluation}

Two approaches are employed to evaluate potential common method bias, namely Harman's single-factor test (Podsakoff and Organ 1986) and the full collinearity approach (Kock 2015). Both assessments indicate an absence of common method bias. Confirmatory tetrad analysis (CTA-PLS) is used to check whether our specifications as reflective measures are appropriate (Gudergan et al. 2008). For all constructs, the reflective model could be confirmed.

To test the model's factor structure, a confirmatory composite analysis is conducted (Hair et al. 2020; Schuberth et al. 2018; Schuberth 2020). SmartPLS 3.3.2 is used for calculation (Ringle et al. 2015). The PLS algorithm is set to a maximum of 300 iterations, a stop criterion of $10^{-7}$, and a path weighting scheme. The covariance matrix is provided in "Appendix B". All indicators' outer loadings should exceed a threshold of 0.708 (Hair et al. 2019), which is the case for all manifest variables except for PI3, SOA3, and SOC4. Construct validity and reliability are checked, drawing on composite reliability (CR) and the average variance extracted (AVE). For all constructs, the thresholds of 0.70 for CR and of 0.50 for AVE are met (Hair et al. 2019). Hence, we decided to retain PI3, SOA3, and SOC4 (see, e.g., Hair et al. 2016). Table 2 displays the assessment of our constructs.

Discriminant validity is checked using an examination of cross-loadings, the Fornell-Larcker criterion, and the heterotrait-monotrait ratio (HTMT) (Henseler et al. 2015b). The HTMT ratios are provided in Table 2; evaluations of the FornellLarcker criterion and cross-loadings are displayed in "Appendices C and D". As can be observed, HTMT rations are within the recommended range below 0.85 for all pairs except for PR-BI, which yields a value of 0.859 . Albeit above the conservative threshold, the literature suggests a second, more liberal anchor of 0.90 (Henseler et al. 2015a). A bootstrapping procedure using 10,000 draws further corroborates discriminant validity, showing that all 95 and 99 percent confidence intervals' upper borders are far off the null value of 1 (Henseler et al. 2015a). Discriminant validity could be established, and hence, the assessment of the outer model is complete. 
Table 3 HTMT ratios

\begin{tabular}{lllllllll}
\hline & PE & EE & HM & PI & PR & BI & SOC & SOA \\
\hline PE & & & & & & & & \\
EE & 0.419 & & & & & & & \\
HM & 0.658 & 0.566 & & & & & & \\
PI & 0.173 & 0.355 & 0.273 & & & & & \\
PR & 0.798 & 0.464 & 0.789 & 0.250 & & & & \\
BI & 0.770 & 0.493 & 0.738 & 0.363 & 0.859 & & & \\
SOC & 0.064 & 0.102 & 0.119 & 0.213 & 0.118 & 0.112 & & \\
SOA & 0.091 & 0.132 & 0.098 & 0.350 & 0.090 & 0.083 & 0.401 & \\
\hline
\end{tabular}

Table 4 Explanatory power. Interpretation adopted from Hair et al. (2019)

\begin{tabular}{llllll}
\hline Construct & $\mathrm{R}^{2}$ value & $\mathrm{R}^{2}$ adjusted & Interpretation & $\mathrm{Q}^{2}$ value & Interpretation \\
\hline $\mathrm{PE}$ & 0.562 & 0.557 & Moderate & 0.449 & Medium to large relevance \\
$\mathrm{EE}$ & 0.183 & 0.174 & Weak & 0.090 & Small relevance \\
$\mathrm{HM}$ & 0.545 & 0.535 & Moderate & 0.403 & Medium to large relevance \\
$\mathrm{BI}$ & 0.731 & 0.705 & Substantial & 0.509 & Large relevance \\
\hline
\end{tabular}

\subsection{Inner model evaluation}

The evaluation of the inner model begins with a check for potential collinearity problems. Variance inflation factors (VIFs) are employed for this purpose, with values below 3 indicating an absence of collinearity issues (Hair et al. 2019). The highest VIF is 1.940 , and consequently, we assume that collinearity is not a threat to our model. In the next step, the coefficient of determination $\left(\mathrm{R}^{2}\right)$ is used to assess the model's explanatory power. The highest value was calculated for BI $\left(R^{2}=0.731\right)$, followed by $P E\left(R^{2}=0.562\right)$. HM could be explained with a similar fit $\left(\mathrm{R}^{2}=0.545\right)$. For $\mathrm{EE}$, the explanatory power was reasonably low, yielding an $\mathrm{R}^{2}$ of 0.183 . Through blindfolding, $\mathrm{Q}^{2}$ values were derived. All values are greater than zero and indicate relevance (Hair et al. 2019), yielding values of 0.509 for BI, 0.449 for PE, 0.090 for EE, and 0.403 for HM. In the cases of BI, PE, and $\mathrm{HM}$, their respective predictors appear adequate and relevant; however, for EE, the $\mathrm{Q}^{2}$ value is relatively low. Table 3 displays a summarization.

For hypotheses testing, we employ a bootstrapping procedure using 10,000 draws. We evaluate the path relations drawing on path coefficients and $\mathrm{f}^{2}$ measures and interpret $95 \%$ confidence intervals as compatibility intervals, i.e., a span of values that are compatible with our empirical data. Table 4 summarizes the results.

As Table 5 displays, most hypotheses could be corroborated. No evidence was found for EE's impact on BI, and three moderating effects: SOC did not statistically significantly influence the EE-BI relation, and the impact of SOA on PR-PE and PR-EE was also not striking. Regarding the research model's dual-purpose core, PE 


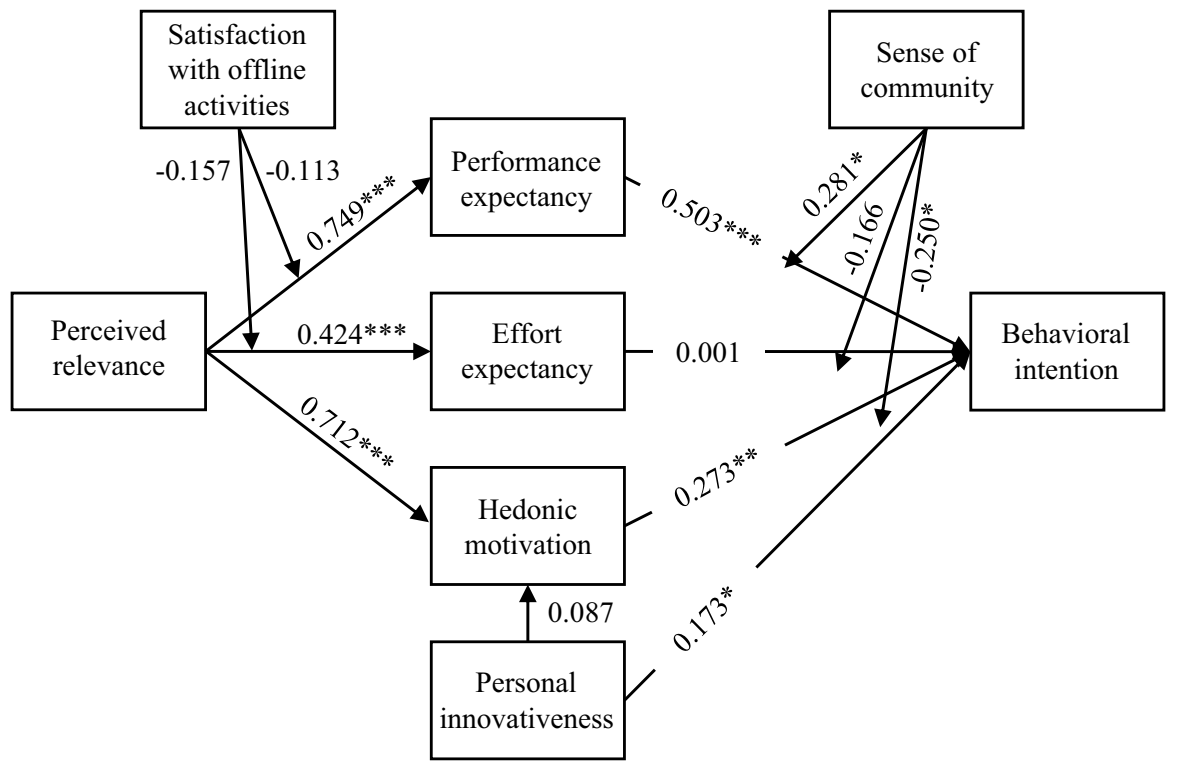

Fig. 2 PLS result. Values indicate path coefficient; ${ }^{*} p<0.05 ; * * p<0.01 ; * * * p<0.001$

yielded a large effect on BI $\left(\mathrm{f}^{2}=0.521\right)$, followed by HM with a medium influence $\left(\mathrm{f}^{2}=0.143\right)$. PI exhibited a small but significant impact on BI.

Considering the formation of PE, EE, and HM, PR was found to be a substantial influence with a very large impact on PE $\left(\mathrm{f}^{2}=1.321\right)$, a medium effect on EE $\left(f^{2}=0.229\right)$, and another large influence on HM $\left(f^{2}=1.046\right)$. The remaining moderator relations, SOC's influence on the PE-BI and the HM-BI link, were both found to exhibit medium effects $\left(\mathrm{f}^{2}=0.224\right.$ and 0.187 , respectively). However, the direction of the moderating effect on the HM-BI relation contradicts our hypothesis, yielding a negative sign. Consequently, the larger coworkers' sense of community is, the smaller the impact of HM on their intention to use the matchmaking tool. Figure 2 presents a succinct summary of our findings.

\subsection{Necessary condition analysis}

Following the recommendations by Richter et al. (2020), a necessary condition analysis (NCA)was conducted (Dul 2016a). Latent variable scores are exported from the PLS model and used as input. In contrast to other approaches such as fsQCA (Ragin 2009), which allow an examination of necessity in kind (i.e., yes or no), NCA provides further insights into each condition's degree of constraint that is imposed on the outcome (Dul 2016b). Consequently, PLS and NCA may be used as complementary analyses: while PLS ensures the validity and reliability of the measurement model and gives information about each variable's sufficiency in terms of path coefficients, NCA reveals potential necessity qualities that need to be considered when deriving theoretical or practical implications (Richter et al. 2020). 
Table 5 Hypotheses testing

\begin{tabular}{|c|c|c|c|c|c|c|}
\hline \multicolumn{4}{|c|}{ Hypothesis } & \multirow{2}{*}{$\begin{array}{l}\text { Path coefficient ( } f^{2} \text { value) } \\
0.503(0.521)\end{array}$} & \multirow{2}{*}{$\begin{array}{l}95 \text { percent con- } \\
\text { fidence interval } \\
(\mathrm{BCa})\end{array}$} & \multirow{2}{*}{$\begin{array}{r}\text { T-value ( } p \text {-value }) \\
5.999(<0.001)\end{array}$} \\
\hline H1 & $\mathrm{PE}$ & $\rightarrow$ & BI & & & \\
\hline $\mathrm{H} 2$ & $\mathrm{EE}$ & $\rightarrow$ & BI & $0.001(<0.001)$ & {$[-0.162,0.106]$} & $0.018(0.986)$ \\
\hline $\mathrm{H} 3$ & HM & $\rightarrow$ & BI & $0.273(0.143)$ & {$[0.113,0.453]$} & $2.889(0.004)$ \\
\hline $\mathrm{H} 4 \mathrm{a}$ & PI & $\rightarrow$ & BI & $0.173(0.090)$ & {$[0.011,0.319]$} & $2.436(0.015)$ \\
\hline $\mathrm{H} 4 \mathrm{~b}$ & PI & $\rightarrow$ & HM & $0.087(0.016)$ & {$[-0.089,0.240]$} & $1.059(0.290)$ \\
\hline $\mathrm{H} 5 \mathrm{a}$ & PR & $\rightarrow$ & $\mathrm{PE}$ & $0.749(1.321)$ & {$[0.652,0.837]$} & $15.746(<0.001)$ \\
\hline $\mathrm{H} 5 \mathrm{~b}$ & PR & $\rightarrow$ & $\mathrm{EE}$ & $0.424(0.229)$ & {$[0.240,0.577]$} & $4.614(<0.001)$ \\
\hline $\mathrm{H} 5 \mathrm{c}$ & PR & $\rightarrow$ & HM & $0.712(1.046)$ & {$[0.608,0.802]$} & $14.509(<0.001)$ \\
\hline H6a & SOC & $\rightarrow$ & $\mathrm{PE} \rightarrow \mathrm{BI}$ & $0.281(0.224)$ & {$[0.160,0.438]$} & $2.430(0.015)$ \\
\hline $\mathrm{H} 6 \mathrm{~b}$ & SOC & $\rightarrow$ & $\mathrm{EE} \rightarrow \mathrm{BI}$ & $-0.166(0.072)$ & {$[-0.381,0.045]$} & $1.035(0.301)$ \\
\hline $\mathrm{H} 6 \mathrm{c}$ & SOC & $\rightarrow$ & $\mathrm{HM} \rightarrow \mathrm{BI}$ & $-0.250(0.187)$ & {$[-0.394,-0.032]$} & $2.280(0.023)$ \\
\hline $\mathrm{H} 7 \mathrm{a}$ & SOA & $\rightarrow$ & $\mathrm{PR} \rightarrow \mathrm{PE}$ & $-0.113(0.026)$ & {$[-0.279,0.325]$} & $0.585(0.559)$ \\
\hline $\mathrm{H} 7 \mathrm{~b}$ & SOA & $\rightarrow$ & $\mathrm{PR} \rightarrow \mathrm{EE}$ & $-0.157(0.024)$ & {$[-0.276,0.443]$} & $0.738(0.461)$ \\
\hline
\end{tabular}

Table 6 NCA results. The observations column indicates the number of cases located above the ceiling line

\begin{tabular}{lllclr}
\hline Condition & Observations & Accuracy $(\%)$ & $p$-accuracy & Effect size d & $p$-value \\
\hline PE & 4 & 95.7 & $<0.001$ & 0.230 & $<0.001$ \\
EE & 2 & 97.8 & $<0.001$ & 0.349 & $<0.001$ \\
HM & 3 & 96.7 & $<0.001$ & 0.274 & $<0.001$ \\
PI & 4 & 95.7 & 0.006 & 0.133 & 0.112 \\
\hline
\end{tabular}

To perform NCA, XY plots containing the data points are drawn for each condition-outcome combination (with the condition on the horizontal and the outcome on the vertical axis), and a ceiling line is drawn above the scatterplot (Dul 2016a). The area atop, i.e., the ceiling zone, is an empty sector that describes values of the outcome that are constrained by the condition. The larger this zone is, the more substantial the effect of the necessary condition (Dul 2016b). For the study at hand, we use ceiling regression - free disposal hull (CR-FDH). Our outcome of interest is $\mathrm{BI}$, and our conditions are the variables from our research model that yield a direct impact: PE, EE, HM, and PI. To assess the NCA results for their statistical significance, we carried out a bootstrapping procedure with 10,000 draws. The results are presented in Table 6 .

To gain more detailed insights, the results can be presented using the bottleneck technique (Dul 2016a), which is displayed in Table 7. As can be observed, all four conditions are necessary and impose moderate to reasonably strong constraints on the outcome. PI yields the weakest restrictions and becomes only necessary for high values of $\mathrm{BI}$; still, to allow the full range of BI to unfold, the 
Table 7 Bottleneck table

\begin{tabular}{lllll}
\hline $\mathrm{Y}$ & $\mathrm{EE}$ & $\mathrm{HM}$ & $\mathrm{PE}$ & $\mathrm{PI}$ \\
\hline 0 & $\mathrm{NN}$ & $\mathrm{NN}$ & $\mathrm{NN}$ & $\mathrm{NN}$ \\
10 & 0.3 & 3.0 & $\mathrm{NN}$ & $\mathrm{NN}$ \\
20 & 8.8 & 9.1 & $\mathrm{NN}$ & $\mathrm{NN}$ \\
30 & 17.4 & 15.2 & 5.0 & $\mathrm{NN}$ \\
40 & 26.0 & 21.3 & 12.9 & $\mathrm{NN}$ \\
50 & 34.5 & 27.4 & 20.7 & $\mathrm{NN}$ \\
60 & 43.1 & 33.4 & 28.6 & 6.4 \\
70 & 51.7 & 39.5 & 36.5 & 16.5 \\
80 & 60.2 & 45.6 & 44.4 & 26.5 \\
90 & 68.8 & 51.7 & 52.3 & 36.6 \\
100 & 77.4 & 57.8 & 60.2 & 46.6 \\
\hline $\mathrm{Y}$ & & & &
\end{tabular}

Y denotes the outcome, i.e., the occurrence of BI. All values in percent

requirements of PI quickly increase to about a third (36.6\%) and a half (46.6\%), respectively. HM is the second-to-last condition; however, note that all constraints become rather strict for high outcome values. For relatively moderate BI values (i.e., around 50\%), about a third of HM needs to be in place. PE plays an even more substantial role, rising to $60.2 \%$ for the full range of BI. Finally, EE is the condition sticking out the most, requiring half of its range for moderate to high values of BI and increasing to two-thirds and three-quarters to allow BI to unfold in its entirety.

In the last step of our analysis, we combine the results from PLS-SEM (i.e., information about each variable's sufficiency) and NCA (i.e., information about each variable's necessity). Table 8 summarizes our findings. We also included the three remaining calculations for $\mathrm{H} 4 \mathrm{~b}, \mathrm{H} 5 \mathrm{a}, \mathrm{H} 5 \mathrm{~b}$, and $\mathrm{H} 5 \mathrm{c}$; however, these are not the focus of our analysis. Overall, the fruitfulness of combining PLSSEM and NCA could be proven. For example, a focus on PLS-SEM would have resulted in EE being treated as irrelevant; however, this conclusion is only valid in terms of a sufficient condition. Our NCA reveals that EE is indeed a necessary condition and yields a large effect on BI. In total, all four predictors of BI (i.e., PE, EE, HM, and PI) were identified as being necessary in degree with varying constraints. Besides EE, the most substantial effect is imposed by HM, followed by PE and then PI. In terms of sufficiency, PE exhibits the most substantial effect on BI, while HM and PI play a subordinate role. EE does not yield any striking impact at all. SOA was found not to have a moderating influence. For SOC, two out of three postulated effects could be verified: it positively moderates the impact of PE on BI and has a negative effect on HM's influence on $\mathrm{BI}$. The role of PR is striking: the variable is necessary for PE and HM, yielding medium effects, and sufficient with large effects for PE and HM, and a medium effect for EE (Table 8). 
Table 8 Total result summary

\begin{tabular}{|c|c|c|c|c|c|}
\hline \multicolumn{4}{|c|}{ Hypothesis } & \multirow{2}{*}{$\begin{array}{l}\text { Necessary condition } \\
\text { Yes; medium effect }(d=0.230)\end{array}$} & \multirow{2}{*}{$\begin{array}{l}\text { Sufficient condition } \\
\text { Yes; large effect }\left(\mathrm{f}^{2}=0.521\right)\end{array}$} \\
\hline $\mathrm{H} 1$ & PE & $\rightarrow$ & BI & & \\
\hline $\mathrm{H} 2$ & $\mathrm{EE}$ & $\rightarrow$ & BI & Yes; large effect $(\mathrm{d}=0.349)$ & No \\
\hline H3 & $\mathrm{HM}$ & $\rightarrow$ & BI & Yes; medium effect $(\mathrm{d}=0.274)$ & Yes; medium effect $\left(\mathrm{f}^{2}=0.143\right)$ \\
\hline $\mathrm{H} 4 \mathrm{a}$ & PI & $\rightarrow$ & BI & Yes; medium effect $(\mathrm{d}=0.133)$ & Yes; small effect $\left(\mathrm{f}^{2}=0.090\right)$ \\
\hline $\mathrm{H} 4 \mathrm{~b}$ & PI & $\rightarrow$ & $\mathrm{HM}$ & No & Yes; small effect $\left(f^{2}=0.074\right)$ \\
\hline $\mathrm{H} 5 \mathrm{a}$ & PR & $\rightarrow$ & $\mathrm{PE}$ & Yes; medium effect $(\mathrm{d}=0.251)$ & Yes; large effect $\left(\mathrm{f}^{2}=1.321\right)$ \\
\hline $\mathrm{H} 5 \mathrm{~b}$ & PR & $\rightarrow$ & $\mathrm{EE}$ & No & Yes; medium effect $\left(f^{2}=0.229\right)$ \\
\hline $\mathrm{H} 5 \mathrm{c}$ & PR & $\rightarrow$ & $\mathrm{HM}$ & Yes; medium effect $(\mathrm{d}=0.235)$ & Yes; large effect $\left(\mathrm{f}^{2}=1.046\right)$ \\
\hline H6a & SOC & $*$ & PE BI & No & Yes; medium effect $\left(f^{2}=0.224\right)$ \\
\hline $\mathrm{H} 6 \mathrm{~b}$ & SOC & $*$ & EE BI & No & No \\
\hline $\mathrm{H} 6 \mathrm{c}$ & SOC & $*$ & HM BI & No & Yes; medium effect $\left(f^{2}=0.187\right)$ \\
\hline $\mathrm{H} 7 \mathrm{a}$ & SOA & $*$ & PR PE & No & No \\
\hline $\mathrm{H} 7 \mathrm{~b}$ & SOA & $*$ & PR EE & No & No \\
\hline
\end{tabular}

\section{Discussion}

Consistent with the extant literature, coworkers' perception of utilitarian benefits was found to yield the most substantial impact on their intention to use a matchmaking tool in terms of sufficiency (Kopplin 2020). Personal innovativeness, however, was also identified as an influential driver, which contrasts with these previous findings. The study at hand employed a UTAUT2-based framework, as opposed to TAM in the extant literature. Hence, a difference might occur due to the modifications of the structural model: as opposed to the TAM framework, where behavioral beliefs (except for perceived usefulness) only directly influence an individual's attitude towards using a particular technology, UTAUT2 postulates a direct linkage between technology acceptance factors and behavioral intention to use (Venkatesh et al. 2012). As our sample was also drawn from independent CWS, we believe that a systematic difference between both studies is rather unlikely.

Compared to the initial UTAUT2, we find an increased influence of PE $\left(\mathrm{f}^{2}: 0.52\right.$ versus 0.21 in the original model $)^{2}$ and a substantially decreased impact of EE $\left(\mathrm{f}^{2}:<0.01\right.$ versus 0.16$)$. HM shows a similar slightly lower effect in our context $\left(\mathrm{f}^{2}: 0.14\right.$ versus 0.23 ). Hence, we report a stronger focus on utilitarian aspects for matchmaking tools in CWS. The apparent difference regarding EE is striking at first; however, many studies find effort considerations to play only a minor role. Software applications both for computers and smartphones have been around for many years and rely on similar icons and interaction mechanisms, which might explain the finding for EE. At this point, it is important to bear in mind that PLS detects sufficient conditions. Our complementary NCA reveals that EE indeed yields a large effect and

\footnotetext{
${ }^{2}$ As the results in the UTAUT2 paper provide two decimals, (Venkatesh et al. 2012), we do the same for readability purposes.
} 
may be considered a necessary condition (Dul 2016b). Additionally, the variables PR and PI proved to exhibit an explanatory value for the context at hand. Moderator analyses showed that SOC is a significant determinant for technology acceptance in CWS, confirming our effort to contextualize our research model (Hong et al. 2014). While SOC is a characteristic property of CWS (Garrett et al. 2017; Gerdenitsch et al. 2016) and thus, narrow in focus, both PI and PR may be readily employed in other contexts for an enhanced understanding of user perceptions of a particular technology, adding insights to the technology acceptance literature through crosscontext applicability (Hong et al. 2014; Venkatesh et al. 2016).

From a necessity perspective, EE's large effect appears plausible for technology in general and matchmaking tools in particular. CWS are designed to enable interaction and communication, and coworkers may leave their desks and talk to others in person (Bouncken et al. 2020b). Consequently, a tool imposing high effort is deemed unattractive. Besides, HM was identified as a necessary condition, consistent with the notion of dual-purpose information systems (see, e.g., Wu and $\mathrm{Lu} 2013$ ). PR shows reasonably mixed results: while the construct is a necessary condition for PE and HM and yields large effects in terms of sufficiency, it is not necessary for EE and only imposes a medium effect in the role of a sufficient condition. This divergence may be explained by the goal alternatives that coworkers may seek to achieve when using a matchmaking tool. Some will use the application as an efficient solution for contacting and, as such, perceive its utilitarian aspects as congruent with their goals. Others stress the hedonic component and consequently view this facet as goal-congruent. EE, in contrast to this dyad, is not a purpose itself but the effort required to utilize the application to achieve a purpose.

Our finding of offline activities (i.e., SOA) not impacting coworkers' perception of digital applications appears counterintuitive at first. We would have assumed that satisfying face-to-face communication opportunities render matchmaking tools irrelevant to at least some degree. Several factors may explain this result: first, daily work patterns may differ between individual coworkers, and hence some potentially helpful contacts may be simply not within the CWS at the current time. Second, it is unlikely to assume that all coworkers excel at networking, and a digital platform may facilitate contact compared to face-to-face interaction at a workshop or breakfast. For example, empirical evidence has been provided that for some coworkers, the potential of participating in a community is more important than actually becoming involved (Garrett et al. 2017), and this potential is tangible in the form of a matchmaking application. Third, it might also be the case that individuals consciously refrain from networking during community events such as lunch or parties, which may be perceived as less business-oriented than pitch sessions or exhibitions, in order not to shift the community's atmosphere from mutual coworking towards viewing each other as customers and business opportunities. Fourth, a matchmaking application allows assessing many profiles at a glance, providing a workflow very different from face-to-face contact, and may be viewed as a self-contained alternative equal to existing forms of social interaction. 
SOC, on the other hand, was confirmed as moderating the influence of PE on BI and the effect of HM on BI. In the case of PE, a positive moderation was expected, as coworkers' perception of social entanglement and belonging increases the likelihood of not only identifying a suitable contact but also being able to benefit from it. For the HM-BI relation, however, SOC reveals an impact contrariwise to our hypothesis, yielding a negative effect. We would have assumed that a high SOC boosts the impact of hedonic factors. Our empirical data suggests, on the contrary, that a lower SOC corresponds to a stronger link, and a higher SOC corresponds to a weaker link. We believe the spirit of coworking can explain this result: a high SOC is likely related to an open and welcoming atmosphere, and in such an environment, the main benefit a matchmaking application may offer is facilitating interaction in terms of efficiency; nevertheless, the community spirit would have allowed for social interaction without the tool, and coworkers who identify with coworking's values (see, e.g., Schuermann 2014) may find enjoyment primarily in personal interaction.

\subsection{Theoretical implications}

The study at hand addressed matchmaking tools in CWS, which has been hardly investigated (Kopplin 2020), although the digital infrastructure is an essential component of coworking (Bouncken et al. 2020b). We advanced the body of knowledge by erecting a coworking-specific technology acceptance model, drawing on wellestablished insights from the literature (see, e.g., Venkatesh et al. 2012). Empirical insights confirmed our structural model, providing insights into the dual-purpose nature of matchmaking tools. Regarding the social composition of CWS, we found relatively stable communities with medium- to long-term memberships, and individual rooms and fixed desks were equally as frequent as hot desks. Hence, coworking in independent CWS is shown to not consist primarily of fast-moving digital nomads but instead of individuals seeking a place where they can get things done-which is consistent with the third-place notion of CWS and the origin of coworking, when knowledge workers sought to break free from isolation at home (Brown 2017; Moriset 2013; Oldenburg 1989). We also provided empirical evidence for coworkers' social orientation (i.e., sense of community) and its impact on technology within the CWS, and coworkers' proclivity to view the coworking environment as a learning opportunity. This fits the notion of gainers (Colleoni and Arvidsson 2015) and novices and mentors (Bouncken and Aslam 2019), respectively. Further, as learning was a common motive regardless of the personal background, we provide support for the suggestion by Waters-Lynch and Potts (2017) to consider learning as the overarching concept of CWS.

Our findings suggest that matchmaking tools may amend CWS as an original class of amenities. They appear not to be influenced by face-to-face interaction. We believe that as digital platforms, accessible anytime and anywhere, they may provide feelings of security and structure. An important motive for coworking is the precarity and atomization of work (Brown 2017; McRobbie 2018), and CWS' community 
may mitigate the impression of being isolated and lost. Matchmaking tools not only visualize the community but make it tangible in a literal sense, as users may swipe, touch, and physically experience the rather abstract concepts of community and collaboration. In a Schelling Point sense (Waters-Lynch and Potts 2017), when individuals perceive that CWS are a focal point to approach, matchmaking tools can be viewed as a technological manifestation of this perception, as they present an impression of the CWS' community, skills, and learning and business opportunities in a structured and comprehensible manner. Consequently, we frame matchmaking tools as a potential remedy for the insight that, frequently, unexpected encounters are fairly scarce (Merkel 2015; Parrino 2015).

Considering technology acceptance research, our analyses show that a combined approach examining necessary as well as sufficient conditions helps increase our understanding of the underlying causal mechanisms. A mere focus on sufficiencyas is the case with regression-based models-would have overlooked essential constraints such as the substantial effect of EE. Awareness of this duality is expected to enable researchers to improve our insights into technology acceptance. The variable SOA further included notions of alternative options the individual coworker may use for socializing. Our data did not provide evidence that the availability of alternatives alters user perceptions of the technology.

\subsection{Practical implications}

Coworkers were found to be willing to employ matchmaking tools for their daily activities in the CWS. While utilitarian factors play the most critical role in their decision-making, HM has been identified as an important driver as well.

Coworkers' satisfaction with offline activities, i.e., events like workshops or pitch sessions, did not have an impact on their perception of matchmaking tools. Consequently, the physical and the digital realm may be viewed as complements rather than as substitutes. As matchmaking tools may establish connections without both coworkers needing to be present within the CWS, they have an advantage over personal interaction in terms of temporal and spatial independence. However, on the other hand, face-to-face contact is much richer and more natural compared to software applications. Hence, coworkers may seek to utilize both to get 'the best of both worlds'. CWS providers should support this entanglement and provide easy-access and up-to-date member databases that are not only lists of coworkers but ready to be analyzed using, for example, matchmaking tools, but also simple queries such as 'who works on a website' and 'who is currently present in my CWS'. Consequently, a matchmaking tool may not be replaced by physical, social events but is a fruitful amenity for a CWS.

As a sense of community was found to have a positive influence on the effect of $\mathrm{PE}$ on intention to use, we may conclude that the digital realm is an enhancement of the physical space that makes the community spirit tangible, and, consequently, this digital layer may also increase coherence and solidarity by providing a who is who of the CWS. 


\subsection{Limitations}

Our study was conducted among German CWS to prevent biases stemming from diversity in the cultural background (see also Bouncken et al. 2018). Consequently, our results need to be treated with care when being applied to other settings. Further, the majority of responses were gathered in CWS located in major cities. Thus, our findings are generally applicable to urban coworking, and further assessment will be helpful to gain insights into coworking in the countryside. Further, the sample sizes can be considered reasonably small. However, PLS-SEM has been found to yield high statistical power even in small-N situations, and, as such, our results appear reasonable. Nevertheless, it is deemed essential to replicate our findings drawing on larger samples. Finally, we opted for independent CWS as our target population, and hence, the results should not be adapted for different types without caution.

\subsection{Future research}

A typical downside of matchmaking, in general, is that the better it works, the more satisfied the users are, and when they found what they were looking for, they are ready to abandon the platform (Kopplin 2020). In the context of coworking, most respondents indicated that they have already been in their current CWS for a rather long time and also intend to stay a member. As most coworkers have not used a matchmaking tool before, it would be a valuable insight whether the implementation of such an application reduced the membership duration. Also, as our sample was reasonably small, we could not examine potential differences between types of CWS users, i.e., utilizers, socializers, and learners, which is a promising opportunity for further investigation. In similar regard, our findings may be challenged by targeting CWS other than independent ones as a population. For example, corporate CWS (Bouncken et al. 2018) would highly benefit from knowledge exchange, inspiration, and innovation.

Besides, both theoretical and empirical studies covering matchmaking tools are rather scarce. Particularly in the context of CWS, coworkers may seek membership for reasons other than searching for a business partner; e.g., they may want to overcome social isolation and achieve a better work-life balance (De Peuter et al. 2017; Orel 2019; Spinuzzi 2007). It might be helpful to develop a typology of matchmaking tools, as different user motives may require different application layouts and functions, and examine the role of these technologies for social and hedonic purposes. Finally, future research should consider other software that can be used in CWS in addition to matchmaking software tools. As mentioned in the introduction, innovation management software could be an example that regulates and possibly simplifies the process of joint innovation in CWS, after matchmaking tools facilitated social connections and elicited serendipity, creativity, and innovation. 


\section{Conclusion}

Concerning the matchmaking tools' embedding within CWS, i.e., their locus in a sociomaterial space, it is essential to note that they are subject to ephemerality (Orlikowski 2007). These applications provide an interface between coworkers, and, in essence, they are a mediator between individuals and, consequently, contingent upon the CWS members, which may vary from day to day. Future research might embrace this dynamic complexity by employing qualitative instruments such as Grounded Theory (Corbin and Strauss 1990), or configurational methods at the intersection of qualitative and quantitative work, such as fuzzy-set qualitative comparative analysis (Ragin 2009). The study at hand sought to provide insights into the matter by combining necessary and sufficient conditions through means of NCA and PLS-SEM, respectively, which might be carried on in future works.

\section{Appendix A: Constructs and items (translated from German)}

See Table 9

\section{Appendix B: Indicator covariance matrix}

See Table 10 


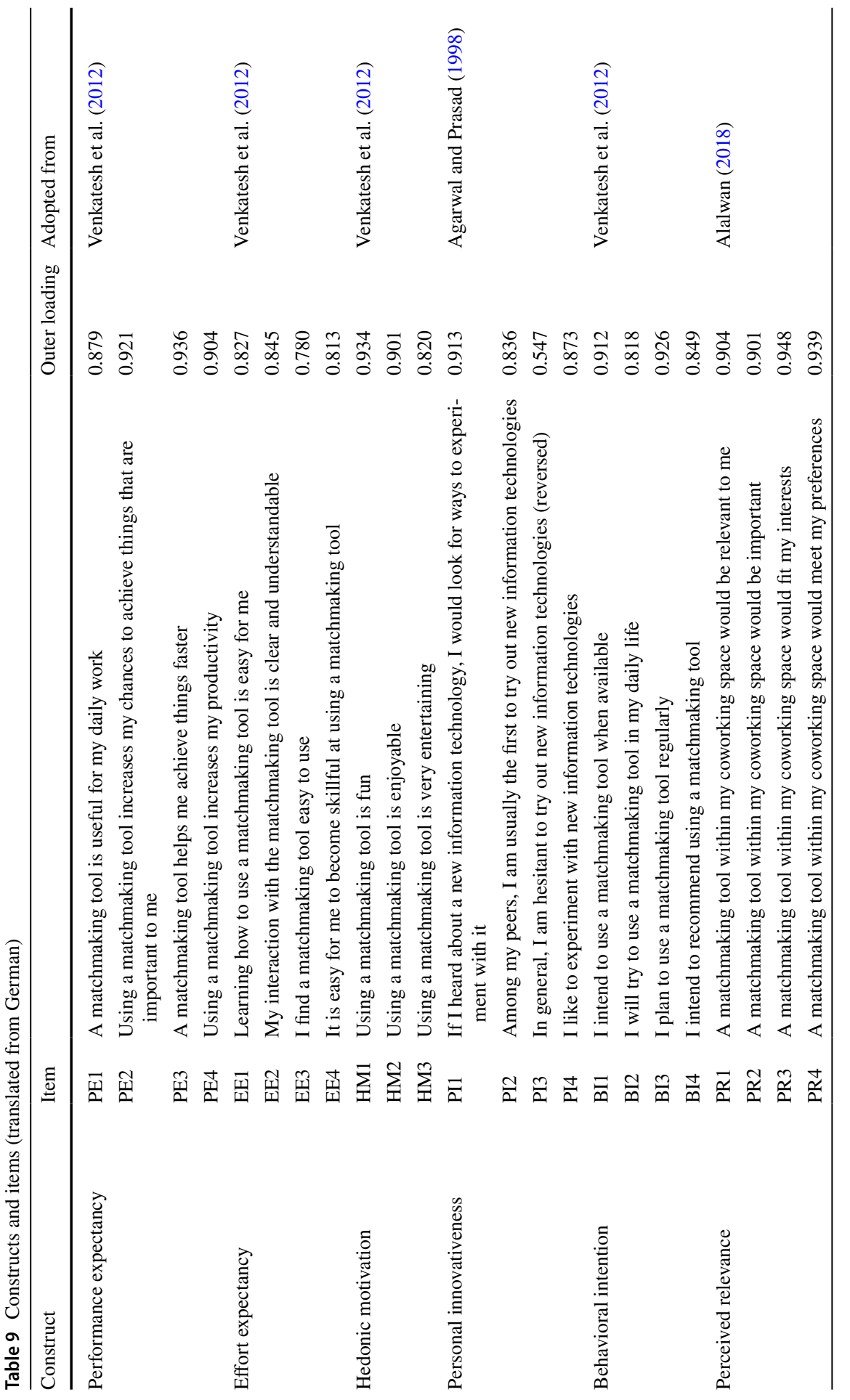




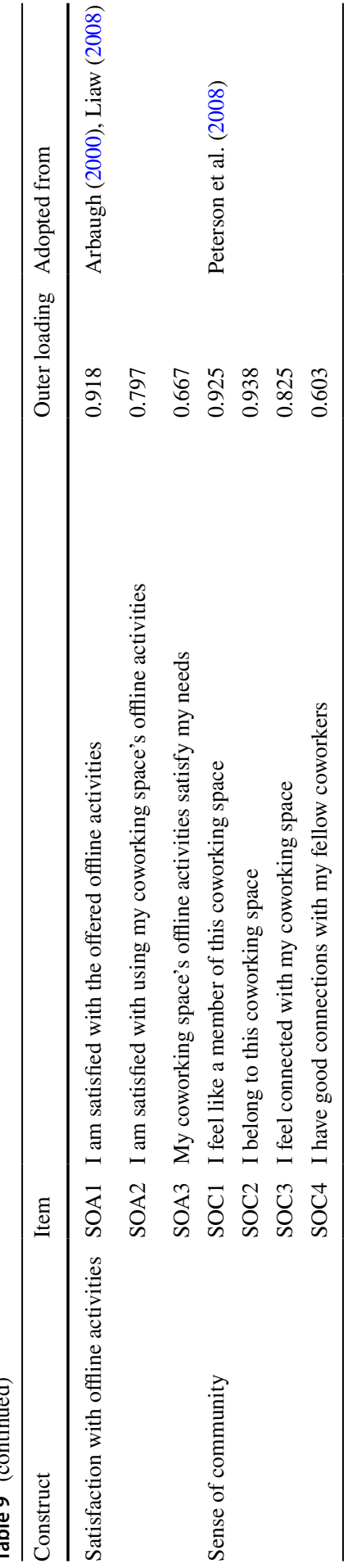




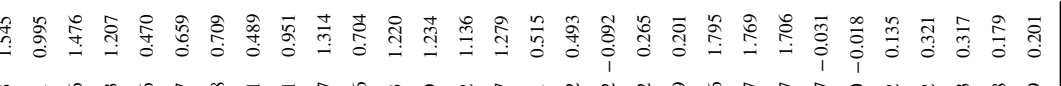

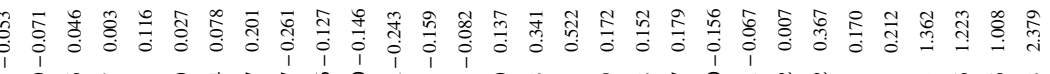

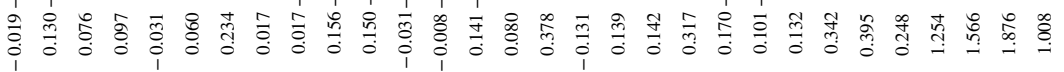

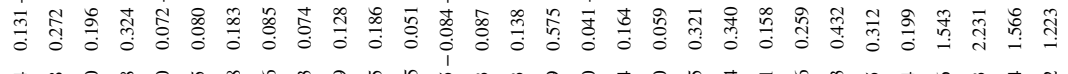

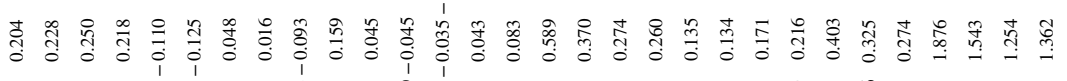

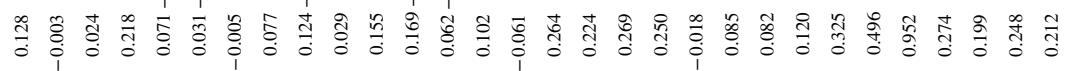

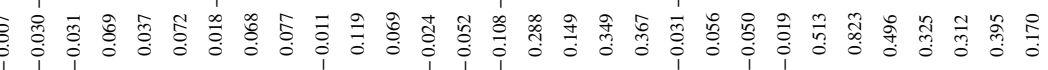

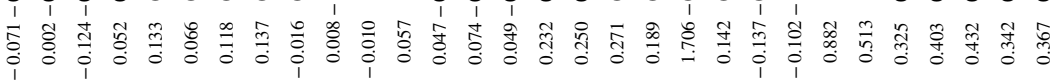

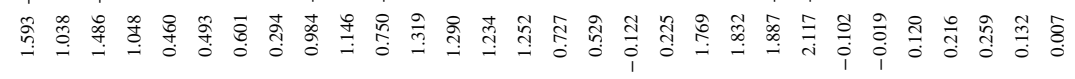

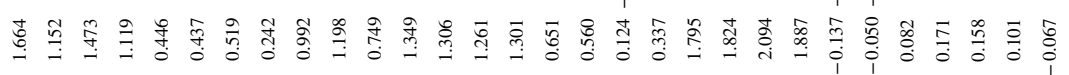

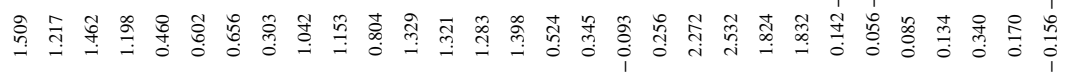

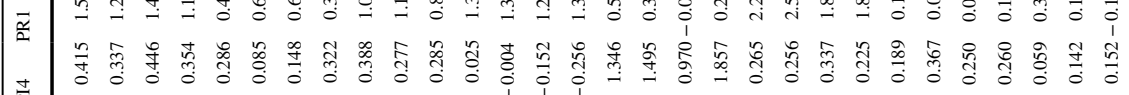

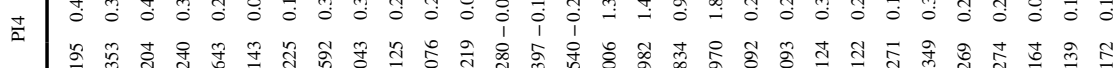

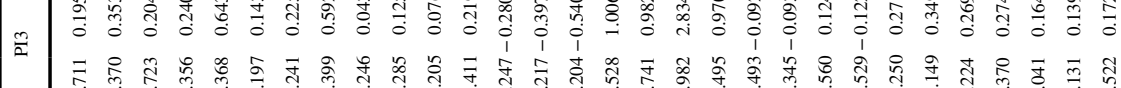
늘

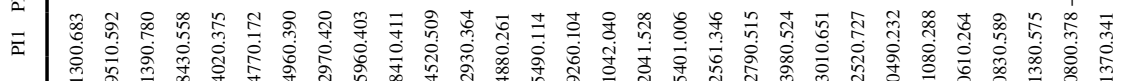

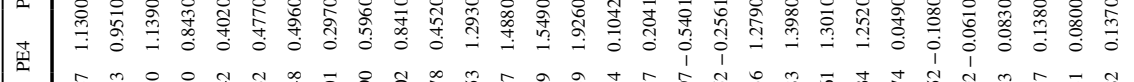

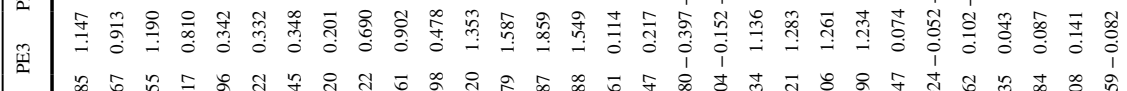

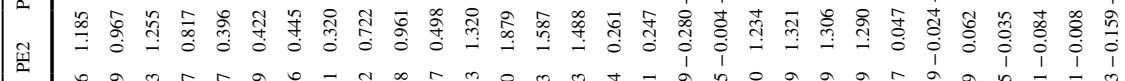

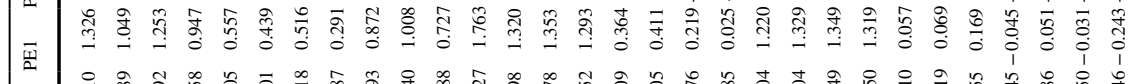

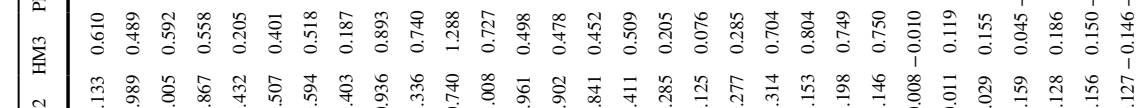

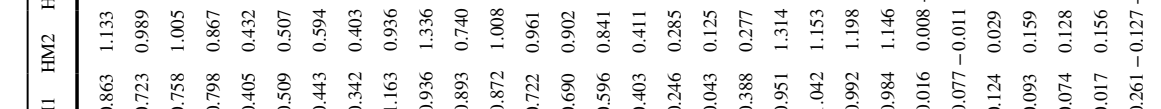

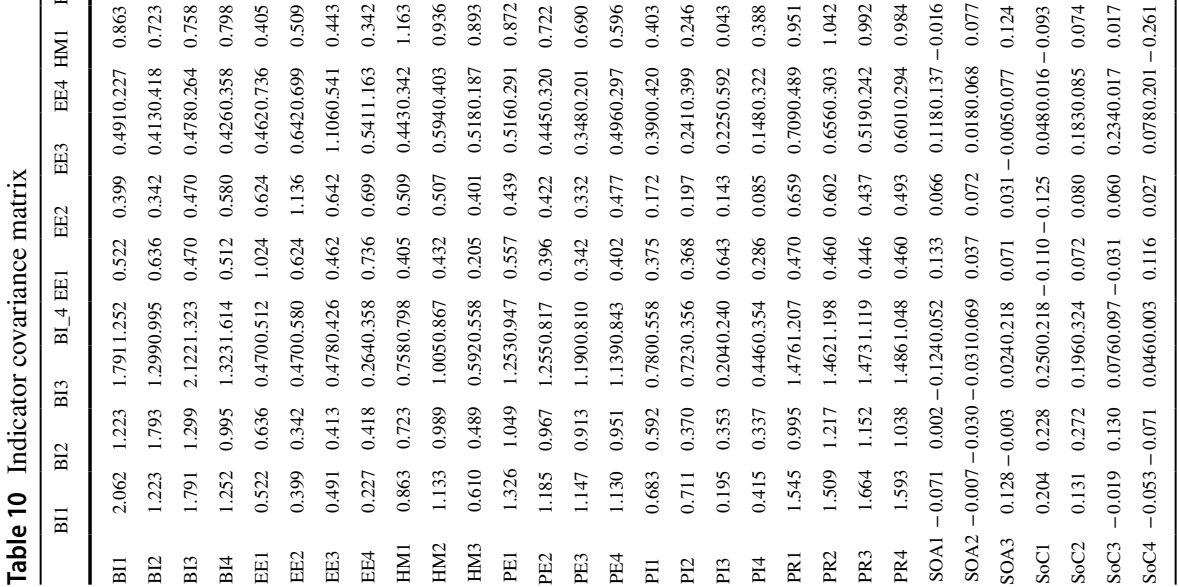




\section{Appendix C: Evaluation of the Fornell-Larcker criterion}

See Table 11

Table 11 Evaluation of the Fornell-Larcker criterion

\begin{tabular}{lllllllll}
\hline & BI & EE & HM & PE & PI & PR & SOA & SOC \\
\hline BI & 0.877 & & & & & & & \\
EE & 0.437 & 0.816 & & & & & & \\
HM & 0.673 & 0.503 & 0.886 & & & & & \\
PE & 0.709 & 0.384 & 0.614 & 0.910 & & & & \\
PI & 0.340 & 0.275 & 0.262 & 0.065 & 0.805 & & & \\
PR & 0.793 & 0.425 & 0.732 & 0.750 & 0.246 & 0.923 & & \\
SOA & 0.005 & 0.124 & 0.039 & 0.046 & 0.248 & 0.003 & 0.800 & \\
SOC & 0.134 & 0.027 & 0.069 & 0.020 & 0.198 & 0.127 & 0.343 & 0.834 \\
\hline
\end{tabular}

\section{Appendix D: Cross-loadings}

See Table 12

Table 12 Cross-loadings

\begin{tabular}{|c|c|c|c|c|c|c|c|c|}
\hline & BI & $\mathrm{EE}$ & $\mathrm{HM}$ & PE & PI & PR & SOA & SOC \\
\hline BI1 & 0.912 & 0.350 & 0.629 & 0.676 & 0.316 & 0.796 & -0.009 & 0.079 \\
\hline BI2 & 0.818 & 0.403 & 0.573 & 0.587 & 0.267 & 0.593 & -0.005 & 0.137 \\
\hline BI3 & 0.926 & 0.349 & 0.557 & 0.672 & 0.339 & 0.732 & -0.062 & 0.109 \\
\hline BI4 & 0.849 & 0.439 & 0.602 & 0.545 & 0.268 & 0.649 & 0.096 & 0.150 \\
\hline EE1 & 0.438 & 0.827 & 0.361 & 0.342 & 0.293 & 0.328 & 0.126 & -0.023 \\
\hline EE2 & 0.349 & 0.845 & 0.451 & 0.317 & 0.112 & 0.370 & 0.070 & -0.013 \\
\hline EE3 & 0.356 & 0.780 & 0.494 & 0.348 & 0.207 & 0.426 & 0.084 & 0.094 \\
\hline EE4 & 0.243 & 0.813 & 0.303 & 0.208 & 0.298 & 0.222 & 0.129 & 0.027 \\
\hline HM1 & 0.605 & 0.466 & 0.934 & 0.544 & 0.246 & 0.665 & 0.042 & -0.002 \\
\hline HM2 & 0.716 & 0.496 & 0.901 & 0.651 & 0.220 & 0.753 & 0.011 & 0.102 \\
\hline HM3 & 0.411 & 0.349 & 0.820 & 0.388 & 0.238 & 0.479 & 0.061 & 0.084 \\
\hline PE1 & 0.713 & 0.412 & 0.668 & 0.879 & 0.158 & 0.711 & 0.083 & 0.001 \\
\hline PE2 & 0.637 & 0.343 & 0.553 & 0.921 & 0.077 & 0.680 & 0.035 & -0.026 \\
\hline PE3 & 0.615 & 0.271 & 0.527 & 0.936 & 0.008 & 0.651 & 0.054 & 0.045 \\
\hline PE4 & 0.605 & 0.361 & 0.472 & 0.904 & -0.020 & 0.680 & -0.007 & 0.057 \\
\hline PI1 & 0.378 & 0.276 & 0.303 & 0.123 & 0.913 & 0.308 & 0.223 & 0.299 \\
\hline PI2 & 0.269 & 0.209 & 0.152 & 0.134 & 0.836 & 0.212 & 0.173 & 0.072 \\
\hline PI3 & 0.122 & 0.273 & 0.049 & -0.111 & 0.547 & -0.019 & 0.216 & 0.096 \\
\hline PI4 & 0.236 & 0.175 & 0.234 & -0.054 & 0.873 & 0.144 & 0.222 & 0.091 \\
\hline PR1 & 0.718 & 0.456 & 0.683 & 0.653 & 0.203 & 0.904 & 0.010 & 0.153 \\
\hline PR2 & 0.701 & 0.383 & 0.644 & 0.677 & 0.176 & 0.901 & 0.089 & 0.112 \\
\hline PR3 & 0.774 & 0.346 & 0.698 & 0.729 & 0.272 & 0.948 & -0.059 & 0.086 \\
\hline PR4 & 0.735 & 0.384 & 0.679 & 0.709 & 0.254 & 0.939 & -0.028 & 0.119 \\
\hline
\end{tabular}


Table 12 (continued)

\begin{tabular}{lrrrrrrrr}
\hline & \multicolumn{1}{l}{ BI } & \multicolumn{1}{l}{ EE } & \multicolumn{1}{l}{ HM } & \multicolumn{1}{l}{ PE } & \multicolumn{1}{l}{ PI } & \multicolumn{1}{l}{ PR } & \multicolumn{1}{l}{ SOA } & \multicolumn{1}{l}{ SOC } \\
\hline SOA1 & -0.030 & 0.141 & -0.005 & 0.049 & 0.193 & -0.029 & $\mathbf{0 . 9 1 8}$ & 0.330 \\
SOA2 & 0.001 & 0.059 & 0.059 & -0.022 & 0.251 & -0.008 & $\mathbf{0 . 7 9 7}$ & 0.282 \\
SOA3 & 0.081 & 0.049 & 0.097 & 0.059 & 0.206 & 0.078 & $\mathbf{0 . 6 6 7}$ & 0.189 \\
SOC1 & 0.136 & -0.040 & 0.029 & 0.005 & 0.243 & 0.112 & 0.332 & $\mathbf{0 . 9 2 5}$ \\
SOC2 & 0.128 & 0.084 & 0.082 & 0.026 & 0.146 & 0.129 & 0.301 & $\mathbf{0 . 9 3 8}$ \\
SOC3 & 0.043 & 0.063 & 0.076 & 0.025 & 0.103 & 0.076 & 0.306 & $\mathbf{0 . 8 2 5}$ \\
SOC4 & -0.010 & 0.075 & -0.117 & -0.049 & 0.161 & -0.001 & 0.241 & $\mathbf{0 . 6 0 3}$ \\
\hline
\end{tabular}

Indicator loadings on their assigned constructs are highlighted in bold

\section{Appendix E: Responses per Coworking Space}

\section{See Table 13}

Table 13 Responses per Coworking Space

\begin{tabular}{|c|c|c|}
\hline Coworking space & Location & $\begin{array}{l}\text { Number of } \\
\text { responses }\end{array}$ \\
\hline Alte kliniken coworking wiesbaden & Wiesbaden & 1 \\
\hline Base co-working münchen & Munich & 5 \\
\hline Betahaus hamburg schanze & Hamburg & 2 \\
\hline Collective.ruhr & Essen & 1 \\
\hline Coworking 4you & Overath & 1 \\
\hline Coworking aachen & Aachen & 1 \\
\hline Cws im.puls & Berlin & 2 \\
\hline Design offices dusseldorf fürst $\&$ friedrich & Dusseldorf & 1 \\
\hline Design offices leipziger platz & Berlin & 2 \\
\hline Edurent regensburg & Regensburg & 3 \\
\hline Engelnest coworking & Berlin & 1 \\
\hline Factory görlitzer park & Berlin & 4 \\
\hline Fleet7 kiel & Kiel & 3 \\
\hline Heartspace & Berlin & 2 \\
\hline Impacthub stuttgart & Stuttgart & 1 \\
\hline Kiezbüro jägerstrasse & Berlin & 1 \\
\hline Kiezbüro neustrelitz & Berlin & 1 \\
\hline Mietwerk potsdam & Potsdam & 1 \\
\hline Nunzig aachen & Aachen & 1 \\
\hline Raumzeit & Hannover & 3 \\
\hline Regus berlin leuchtenfabrik & Berlin & 1 \\
\hline Ruca & Tübungen & 1 \\
\hline Space shack & Berlin & 10 \\
\hline Spaces alte post & Berlin & 3 \\
\hline Spaces düsseldorf andreas quartier & Dusseldorf & 1 \\
\hline Spaces kennedydamm & Dusseldorf & 1 \\
\hline
\end{tabular}


Table 13 (continued)

\begin{tabular}{lll}
\hline Coworking space & Location & $\begin{array}{c}\text { Number of } \\
\text { responses }\end{array}$ \\
\hline St. Oberholz & Berlin & 3 \\
Think space & Berlin & 1 \\
Tinktank & Heidelberg & 1 \\
Trafo braunschwerig & Braunschweig & 4 \\
Tuesday coworking & Berlin & 4 \\
Wework münchen & Munich & 1 \\
Wework oskar von miller münchen & Munich & 1 \\
Wework stralauer allee münchen & Munich & 1 \\
Wework taunusanlage & Frankfurt & 2 \\
Wework warschauer platz & Berlin & 1 \\
Wexelwirken & Reutlingen & 3 \\
Workrepublic düsseldorf medienhafen & Dusseldorf & 4 \\
Workrepublic hamburg neuer dovenhof & Hamburg & 3 \\
Workrepublic hamburg neuer wall & Hamburg & 6 \\
Workrepublic viktualienmarkt & Munich & 1 \\
Worqs aachen & Aachen & 1 \\
\hline Zammwerk chemnitz & Chemnitz & 1 \\
\hline
\end{tabular}

Funding Open Access funding enabled and organized by Projekt DEAL. This research did not receive any specific grant from funding agencies in the public, commercial, or not-for-profit sectors.

Data availability No data is provided.

\section{Declarations}

Conflict of interest The authors disclose that they have no conflict of interest.

Open Access This article is licensed under a Creative Commons Attribution 4.0 International License, which permits use, sharing, adaptation, distribution and reproduction in any medium or format, as long as you give appropriate credit to the original author(s) and the source, provide a link to the Creative Commons licence, and indicate if changes were made. The images or other third party material in this article are included in the article's Creative Commons licence, unless indicated otherwise in a credit line to the material. If material is not included in the article's Creative Commons licence and your intended use is not permitted by statutory regulation or exceeds the permitted use, you will need to obtain permission directly from the copyright holder. To view a copy of this licence, visit http://creativecommons.org/licen ses/by/4.0/.

\section{References}

Agarwal R, Karahanna E (2000) time flies when you're having fun: cognitive absorption and beliefs about information technology usage. MIS Q 24:665-694. https://doi.org/10.2307/3250951

Agarwal R, Prasad J (1998) A conceptual and operational definition of personal innovativeness in the domain of information technology. Inf Syst Res 9:204-215. https://doi.org/10.1287/isre.9.2.204 
Alalwan AA (2018) Investigating the impact of social media advertising features on customer purchase intention. Int J Inf Manag 42:65-77. https://doi.org/10.1016/j.ijinfomgt.2018.06.001

Arbaugh JB (2000) Virtual classroom characteristics and student satisfaction with internet-based MBA courses. J Manag Educ 24:32-54. https://doi.org/10.1177/105256290002400104

Belk R (2014) You are what you can access: sharing and collaborative consumption online. J Bus Res 67:1595-1600. https://doi.org/10.1016/j.jbusres.2013.10.001

Bhattacherjee A, Sanford C (2006) Influence processes for information technology acceptance: an elaboration likelihood model. MIS Q 30:805-825. https://doi.org/10.2307/25148755

Bianchi F, Casnici N, Squazzoni F (2018) Solidarity as a byproduct of professional collaboration: social support and trust in a coworking space. Soc Networks 54:61-72. https://doi.org/10.1016/j. socnet.2017.12.002

Bilandzic M, Foth M (2013) Libraries as coworking spaces: understanding user motivations and perceived barriers to social learning. Library Hi Tech. https://doi.org/10.1108/07378831311329040

Blagoev B, Costas J, Kärreman D (2019) 'We are all herd animals': community and organizationality in coworking spaces. Organization 26:894-916. https://doi.org/10.1177/1350508418821008

Bouncken RB, Aslam MM (2019) Understanding knowledge exchange processes among diverse users of coworking-spaces. J Knowl Manag 23:2067-2085. https://doi.org/10.1108/JKM-05-2018-0316

Bouncken RB, Reuschl AJ (2018) Coworking-spaces: how a phenomenon of the sharing economy builds a novel trend for the workplace and for entrepreneurship. Rev Manag Sci 12:317-334. https://doi.org/10.1007/s11846-016-0215-y

Bouncken RB, Laudien SM, Fredrich V, Görmar L (2018) Coopetition in coworking-spaces: value creation and appropriation tensions in an entrepreneurial space. Rev Manag Sci 12:385-410. https://doi.org/10.1007/s11846-017-0267-7

Bouncken RB, Ratzmann M, Barwinski R, Kraus S (2020a) Coworking spaces: empowerment for entrepreneurship and innovation in the digital and sharing economy. J Bus Res 114:102-110. https://doi.org/10.1016/j.jbusres.2020.03.033

Bouncken RB, Aslam MM, Qiu Y (2020b) Coworking spaces: Understanding, using, and managing sociomateriality. Bus Horiz 64:119-130. https://doi.org/10.1016/j.bushor.2020.09.010

Bouncken RB, Tiberius V (2021) Legitimacy processes and trajectories of co-prosumption services: insights from coworking spaces. J Serv Res (accepted).

Brown J (2017) Curating the "Third Place"? Coworking and the mediation of creativity. Geoforum 82:112-126. https://doi.org/10.1016/j.geoforum.2017.04.006

Campbell DE, Wright RT (2008) Shut-UP I don't care: understanding the role of relevance and interactivity on customer attitudes toward repetitive online advertising. J Electron Commer Res 9:62-76

Capdevila I (2013) Knowledge dynamics in localized communities: coworking spaces as microclusters. Available at SSRN 2414121

Celsi RL, Olson JC (1988) The role of involvement in attention and comprehension processes. J Consum Res 15:210. https://doi.org/10.1086/209158

Colleoni E, Arvidsson A (2015) La partecipazione dei giovani al mercato del lavoro: il ruolo dei coworking space per i giovani freelance. L. Manzo (a cura di), MI Generation. Il Piano Di Governance Delle Politiche Giovanili Della Città Di Milano 2013-2014:141-189

Corbin JM, Strauss A (1990) Grounded theory research: Procedures, canons, and evaluative criteria. Qual Sociol 13:3-21

de Guinea AO, Markus ML (2009) Why break the habit of a lifetime? Rethinking the roles of intention, habit, and emotion in continuing information technology use. MIS Q 33:433-444. https://doi.org/10. 2307/20650303

De Peuter G, Cohen NS, Saraco F (2017) The ambivalence of coworking: On the politics of an emerging work practice. Eur J Cult Stud 20:687-706

DeGuzman GV, Tang AI (2011) Working in the unoffice: A guide to coworking for indie workers, small businesses, and nonprofits. Night Owls Press LLC

Dul J (2016a) Identifying single necessary conditions with NCA and fsQCA. J Bus Res 69:15161523. https://doi.org/10.1016/j.jbusres.2015.10.134

Dul J (2016b) Necessary condition analysis (NCA) logic and methodology of "necessary but not sufficient" causality. Organ Res Methods 19:10-52. https://doi.org/10.1177/1094428115584005

Endres H, Huesig S, Pesch R (2021) Digital innovation management for entrepreneurial ecosystems: services and functionalities as drivers of innovation management software adoption. Rev Manag Sci https://doi.org/10.1007/s11846-021-00441-4 
Fishbein M, Ajzen I (1975) Belief, attitude, intention, and behavior: an introduction to theory and research. Addison-Wesley

Flynn LR, Goldsmith RE (1993) A validation of the Goldsmith and Hofacker innovativeness scale. Educ Psychol Meas 53:1105-1116. https://doi.org/10.1177/0013164493053004023

Gandini A (2015) The rise of coworking spaces: a literature review. ephemera 15:193

Garrett LE, Spreitzer GM, Bacevice PA (2017) Co-constructing a sense of community at work: the emergence of community in coworking spaces. Organ Stud 38:821-842. https://doi.org/10.1177/ 0170840616685354

Gerdenitsch C, Scheel TE, Andorfer J, Korunka C (2016) Coworking spaces: a source of social support for independent professionals. Front Psychol 7:581. https://doi.org/10.3389/fpsyg.2016. 00581

Gudergan SP, Ringle CM, Wende S, Will A (2008) Confirmatory tetrad analysis in PLS path modeling. J Bus Res 61:1238-1249. https://doi.org/10.1016/j.jbusres.2008.01.012

Hair JF, Risher JJ, Sarstedt M, Ringle CM (2019) When to use and how to report the results of PLS-SEM. Eur Bus Rev 33:2-24. https://doi.org/10.1108/EBR-11-2018-0203

Hair JF, Howard MC, Nitzl C (2020) Assessing measurement model quality in PLS-SEM using confirmatory composite analysis. J Bus Res 109:101-110. https://doi.org/10.1016/j.jbusres.2019.11.069

Hair JF, Hult GTM, Ringle C, Sarstedt M (2016) A primer on partial least squares structural equation modeling (PLS-SEM). Sage publications

Harris J, Ives B, Junglas I (2012) IT consumerization: When gadgets turn into enterprise IT tools. MIS Q Exec 11:99-112

Henseler J, Ringle CM, Sarstedt M (2015a) A new criterion for assessing discriminant validity in variance-based structural equation modeling. J Acad Mark Sci 43:115-135. https://doi.org/10.1007/ s11747-014-0403-8

Henseler J, Ringle CM, Sarstedt M (2015b) A new criterion for assessing discriminant validity in variance-based structural equation modeling. J Acad Mark Sci 43:115-135

Hong W, Chan FKY, Thong JYL, Chasalow LC, Dhillon G (2014) A framework and guidelines for context-specific theorizing in information systems research. Inf Syst Res 25:111-136. https://doi.org/ 10.1287/isre.2013.0501

Hu PJ-H, Clark THK, Ma WW (2003) Examining technology acceptance by school teachers: a longitudinal study. Inf Manag 41:227-241. https://doi.org/10.1016/S0378-7206(03)00050-8

Huesig S, Endres H (2019) Exploring the digital innovation process: the role of functionality for the adoption of innovation management software by innovation managers. Eur J Innov Manag 22:302314. https://doi.org/10.1108/EJIM-02-2018-0051

Jackson JD, Yi MY, Park JS (2013) An empirical test of three mediation models for the relationship between personal innovativeness and user acceptance of technology. Inf Manag 50:154-161. https:// doi.org/10.1016/j.im.2013.02.006

Jasperson J, Carter PE, Zmud RW (2005) A comprehensive conceptualization of post-adoptive behaviors associated with information technology enabled work systems. MIS Q 29:525-557. https://doi.org/ $10.2307 / 25148694$

Jung A-R (2017) The influence of perceived ad relevance on social media advertising: an empirical examination of a mediating role of privacy concern. Comput Hum Behav 70:303-309. https://doi.org/10. 1016/j.chb.2017.01.008

Kock N (2015) Common method bias in PLS-SEM: a full collinearity assessment approach. Int J e-Collab (IJeC) 11:1-10. https://doi.org/10.4018/ijec.2015100101

Kopplin CS (2020) Two heads are better than one: matchmaking tools in coworking spaces. Rev Manag Sci 15:1045-1069. https://doi.org/10.1007/s11846-020-00382-4

Liaw S-S (2008) Investigating students' perceived satisfaction, behavioral intention, and effectiveness of e-learning: a case study of the Blackboard system. Comput Educ 51:864-873. https://doi.org/10. 1016/j.compedu.2007.09.005

Limayem M, Hirt SG, Cheung CMK (2007) How habit limits the predictive power of intention: the case of information systems continuance. MIS Q 31:705-737. https://doi.org/10.2307/25148817

Lu J, Yao JE, Yu C-S (2005) Personal innovativeness, social influences and adoption of wireless Internet services via mobile technology. J Strateg Inf Syst 14:245-268

Madden TJ, Ellen PS, Ajzen I (1992) A comparison of the theory of planned behavior and the theory of reasoned action. Pers Soc Psychol Bull 18:3-9

McRobbie A (2018) Be creative: making a living in the new culture industries. Wiley

Merkel J (2015) Coworking in the city. Ephemera 15:121-139 
Merton RK, Barber E (2004) The travels and adventures of serendipity: a study in sociological semantics and the sociology of science. Princeton University Press

Midgley DF, Dowling GR (1978) Innovativeness: the concept and its measurement. J Consum Res 4:229-242. https://doi.org/10.1086/208701

Moriset B (2013) Building new places of the creative economy. The rise of coworking spaces

Oldenburg R (1989) The great good place: Cafés, coffee shops, community centers, beauty parlors, general stores, bars, hangouts, and how they get you through the day. Paragon House Publishers

Orlikowski WJ (2007) Sociomaterial practices: Exploring technology at work. Organ Stud 28:1435-1448

Parrino L (2015) Coworking: assessing the role of proximity in knowledge exchange. Knowl Manag Res Pract 13:261-271. https://doi.org/10.1057/kmrp.2013.47

Peterson NA, Speer PW, McMillan DW (2008) Validation of a brief sense of community scale: confirmation of the principal theory of sense of community. J Commun Psychol 36:61-73. https://doi.org/10. 1002/jcop. 20217

Podsakoff PM, Organ DW (1986) Self-reports in organizational research: problems and prospects. J Manag 12:531-544. https://doi.org/10.1177/014920638601200408

Ragin CC (2009) Qualitative comparative analysis using fuzzy sets (fsQCA). Configurational comparative methods: Qualitative comparative analysis (QCA) and related techniques 51:87-121. https:// doi.org/10.4135/9781452226569.n5

Rese A, Kopplin CS, Nielebock C (2020) Factors influencing members' knowledge sharing and creative performance in coworking spaces. J Knowl Manag 24:2327-2354. https://doi.org/10.1108/ JKM-04-2020-0243

Richter NF, Schubring S, Hauff S, Ringle CM, Sarstedt M (2020) When predictors of outcomes are necessary: Guidelines for the combined use of PLS-SEM and NCA. Ind Manag Data Syst

Ringle CM, Wende S, Becker J-M (2015) SmartPLS 3

Rogers EM, Shoemaker FF (1971) Communication of Innovations; a cross-cultural approach

Schmidt S, Brinks V (2017) Open creative labs: Spatial settings at the intersection of communities and organizations. Creat Innov Manag 26:291-299. https://doi.org/10.1108/JKM-04-2020-0243

Schuberth F (2020) Confirmatory composite analysis using partial least squares: setting the record straight. Rev Manag Sci. https://doi.org/10.1007/s11846-020-00405-0

Schuberth F, Henseler J, Dijkstra TK (2018) Confirmatory composite analysis. Front Psychol 9:2541. https://doi.org/10.3389/fpsyg.2018.02541

Schuermann M (2014) Coworking space: a potent business model for plug " $n$ play and indie workers. epubli

Seo J, Lysiankova L, Ock Y-S, Chun D (2017) Priorities of coworking space operation based on comparison of the hosts and users' perspectives. Sustainability 9:1494. https://doi.org/10.3390/su9081494

Shih H-P (2004) Extended technology acceptance model of Internet utilization behavior. Inf Manag 41:719-729. https://doi.org/10.1016/j.im.2003.08.009

Spinuzzi C (2012) Working alone together: coworking as emergent collaborative activity. J Bus Tech Commun 26:399-441. https://doi.org/10.1177/1050651912444070

Spinuzzi C, Bodrožić Z, Scaratti G, Ivaldi S (2019) "Coworking is about community": but what is "community" in coworking? J Bus Tech Commun 33:112-140. https://doi.org/10.1177/1050651918 816357

Venkatesh V, Morris MG, Davis GB, Davis FD (2003) User acceptance of information technology: toward a unified view. MIS Q 27:425-478. https://doi.org/10.2307/30036540

Venkatesh V, Thong JYL, Xu X (2012) Consumer acceptance and use of information technology: extending the unified theory of acceptance and use of technology. MIS Q 36:157-178. https://doi.org/10. 2307/41410412

Venkatesh V, Thong JYL, Xu X (2016) Unified theory of acceptance and use of technology: a synthesis and the road ahead. J Assoc Inf Syst 17:328-376. https://doi.org/10.2307/41410412

Waters-Lynch J, Potts J (2017) The social economy of coworking spaces: a focal point model of coordination. Rev Soc Econ 75:417-433. https://doi.org/10.1080/00346764.2016.1269938

Wu J, Lu X (2013) Effects of extrinsic and intrinsic motivators on using utilitarian, hedonic, and dualpurposed information systems: a meta-analysis. J Assoc Inf Syst. 14:1. https://doi.org/10.17705/ 1jais.00325

Publisher's Note Springer Nature remains neutral with regard to jurisdictional claims in published maps and institutional affiliations. 